\title{
Sine-Gordon breather form factors and quantum field equations
}

\author{
H. Babujian*† and M. Karowski ${ }^{\ddagger}$ \\ Institut für Theoretische Physik \\ Freie Universität Berlin, \\ Arnimallee 14, 14195 Berlin, Germany
}

November 11, 2018

\begin{abstract}
Using the results of previous investigations on sine-Gordon form factors exact expressions of all breather matrix elements are obtained for several operators: all powers of the fundamental bose field, general exponentials of it, the energy momentum tensor and all higher currents. Formulae for the asymptotic behavior of bosonic form factors are presented which are motivated by Weinberg's power counting theorem in perturbation theory. It is found that the quantum sine-Gordon field equation holds and an exact relation between the "bare" mass and the renormalized mass is obtained. Also a quantum version of a classical relation for the trace of the energy momentum is proven. The eigenvalue problem for all higher conserved charges is solved. All results are compared with perturbative Feynman graph expansions and full agreement is found.
\end{abstract}

PACS: 11.10.-z; 11.10.Kk; 11.55.Ds

Keywords: Integrable quantum field theory, Form factors

\section{Introduction}

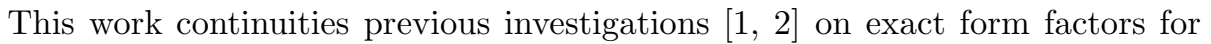
the sine-Gordon alias the massive Thirring model. Some results of the present paper have been published previously [3]. The classical sine-Gordon model is given by the wave equation

$$
\square \varphi(t, x)+\frac{\alpha}{\beta} \sin \beta \varphi(t, x)=0
$$

*Permanent address: Yerevan Physics Institute, Alikhanian Brothers 2, Yerevan, 375036 Armenia.

†e-mail: babujian@lx2.yerphi.am, babujian@physik.fu-berlin.de

¥e-mail: karowski@physik.fu-berlin.de 
Since Coleman [4] found the wonderful duality between the quantum sineGordon and the massive Thirring model a great deal of effort have been made to understand this quantum field theoretic model. The present article is a further contribution in this direction. The main new results are:

1. Using Weinberg's power counting theorem we prove in perturbation theory that matrix elements of exponentials of a bose field satisfy a 'cluster property' in momentum space. We use this as a characterizing property for exponentials of bose fields

2. In 2] we introduced the concept of 'p-functions' which belong to local operators for the sine-Gordon solitons (see also [5, 6]). Here we formulate that concept for the sine-Gordon breathers.

3. We investigate the higher conservation laws which are typical for integrable quantum field theories. Hereby we correct a mistake in the literature (see the footnote 7 ).

4. We prove the quantum field equation of motion. Thus we derive independently the matrix elements of the operators $\varphi$ and $: \sin \beta \varphi$ : and show that they satisfy the field equation (after a finite mass renormalization). Hereby we again correct some mistakes in the literature (see the footnote 8).

In addition we also recall some known formulae in order to present a more complete picture of the sine-Gordon breather form factors. The sine-Gordon model alias the massive Thirring model describes the interaction of several types of particles: solitons, anti-solitons alias fermions and anti-fermions and a finite number of charge-less breathers, which may be considered as bound states of solitons and anti-solitons. Integrability of the model implies that the n-particle S-matrix factorizes into two particle S-matrices.

The "bootstrap" program for integrable quantum field theoretical models in $1+1$ dimensions starts as the first step with the calculation of the S-matrix. Here (see e.g. [7, 8]) our starting point is the two particle sine-Gordon S-matrix for the scattering of fundamental bosons (lowest breathers) [9]

$$
S(\theta)=\frac{\sinh \theta+i \sin \pi \nu}{\sinh \theta-i \sin \pi \nu}
$$

The pole of $S(\theta)$ at $\theta=i \pi \nu$ belongs to the second breather $b_{2}$ as a breatherbreather bound state. The parameter $\nu$ is related to the sine-Gordon and the massive Thirring model coupling constant by

$$
\nu=\frac{\beta^{2}}{8 \pi-\beta^{2}}=\frac{\pi}{\pi+2 g}
$$

where the second equation is due to Coleman [4.

\footnotetext{
${ }^{1}$ This S-matrix element has been discussed before in 10, 11.
} 
As a second step of the "bootstrap" program off-shell quantities as arbitrary matrix elements of local operators

$$
{ }^{\text {out }}\left\langle p_{m}^{\prime}, \ldots, p_{1}^{\prime}|\mathcal{O}(x)| p_{1}, \ldots, p_{n}\right\rangle^{\text {in }}
$$

are obtained by means of the "form factor program" from the S-matrix as an input. Form factors for integrable model in $1+1$ dimensions were first investigated by Vergeles and Gryanik [10] for the sinh-Gordon model and by Weisz $[12]^{2}$ for the sine-Gordon model. The 'form factor program' was formulated in [14. 15] where the concept of generalized form factors was introduced. In that article consistency equations were formulated which are expected to be satisfied by these objects. Thereafter this approach was developed further and studied in the context of several explicit models by Smirnov [16] who proposed the form factor equations (see below) as extensions of similar formulae in the original article [14]. Further publications on form factors and in particular on sine-Gordon and sinh-Gordon form factors are [17] 28 . Smirnov's approach in 17] is similar to the one used in the present article (see section 6). Also there is a nice application [29, 30] of form factors in condensed matter physics for example for the one dimensional Mott insulators.

Let $\mathcal{O}(x)$ be a local operator. The generalized form factors $\mathcal{O}_{n}\left(\theta_{1}, \ldots, \theta_{n}\right)$ are defined by the vacuum - n-lowest breather matrix elements

$$
\left\langle 0|\mathcal{O}(x)| p_{1}, \ldots, p_{n}\right\rangle^{i n}=e^{-i x\left(p_{1}+\cdots+p_{n}\right)} \mathcal{O}_{n}\left(\theta_{1}, \ldots, \theta_{n}\right), \quad \text { for } \quad \theta_{1}>\cdots>\theta_{n}
$$

where the $\theta_{i}$ are the rapidities of the particles $p_{i}^{\mu}=m\left(\cosh \theta_{i}, \sinh \theta_{i}\right)$. In the other sectors of the variables the functions $\mathcal{O}_{n}\left(\theta_{1}, \ldots, \theta_{n}\right)$ are given by analytic continuation with respect to the $\theta_{i}$. General matrix elements are obtained from $\mathcal{O}_{n}(\underline{\theta})$ by crossing which means in particular the analytic continuation $\theta_{i} \rightarrow$ $\theta_{i} \pm i \pi$.

In 14 one of the present authors (M.K.) and Weisz showed that for the case of a diagonal S-matrix the n-particle form factor may be written as

$$
\mathcal{O}_{n}(\underline{\theta})=K_{n}^{\mathcal{O}}(\underline{\theta}) \prod_{1 \leq i<j \leq n} F\left(\theta_{i j}\right)
$$

where $\theta_{i j}=\left|\theta_{i}-\theta_{j}\right|$ and $F(\theta)$ is the two-particle form factor (see section 2). The $\mathrm{K}$-function is an even $2 \pi i$ periodic meromorphic function. In [2] we presented a general formula for soliton anti-soliton form factors in terms of an integral representation. Using the bound state fusion method we derived the general soliton-breather and pure breather form factor formula which we will investigate in this article in more detail. In particular for the case of lowest breathers the

\footnotetext{
${ }^{2}$ Similar results were obtained by Zamolodchikov 13 .
} 
K-function turns out to be of the form ${ }^{3}$

$$
K_{n}^{\mathcal{O}}(\underline{\theta})=\sum_{l_{1}=0}^{1} \cdots \sum_{l_{n}=0}^{1}(-1)^{l_{1}+\cdots+l_{n}} \prod_{1 \leq i<j \leq n}\left(1+\left(l_{i}-l_{j}\right) \frac{i \sin \pi \nu}{\sinh \theta_{i j}}\right) p_{n}^{\mathcal{O}}(\underline{\theta}, \underline{l}) .
$$

The breather p-function $p_{n}^{\mathcal{O}}\left(\theta_{1}, \ldots, \theta_{n}, l_{1}, \ldots, l_{n}\right)$ encodes the dependence on the operator $\mathcal{O}(x)$. It is obtained from the solitonic p-function $p_{s o l, 2 n}^{\mathcal{O}}\left(\tilde{\theta}_{1}, \ldots, \tilde{\theta}_{2 n}\right.$; $z_{1}, \ldots, z_{n}$ ) (see [2]) by setting $\tilde{\theta}_{2 i-1}=\theta_{i}+\frac{1}{2} i u^{(1)}, \tilde{\theta}_{2 i}=\theta_{i}-\frac{1}{2} i u^{(1)}$ and $z_{j}=$ $\theta_{j}-\frac{i \pi}{2}\left(1-(-1)^{l_{j}} \nu\right)$ with the fusion angle $u^{(1)}=\pi(1-\nu)$. In [2] we proposed the solitonic p-functions for several local operators. In this way we obtained all breather p-functions which are some sort of decedents of solitonic p-functions i.e. we just used the solitonic ones in the bound state points. In the present article we will take a somewhat different point of view. We will obtain a wider class of p-functions corresponding to local operators with respect to the breather field, including also operators which are non-local with respect to the solitonic field. The alternative point of view is the following: As already mentioned, it has been shown in [14 that a form factor of $\mathrm{n}$ fundamental bosons (lowest breathers) is of the form (1) where the K-function $K_{n}^{\mathcal{O}}(\underline{\theta})$ is meromorphic, symmetric and periodic (under $\theta_{i} \rightarrow \theta_{i}+2 \pi i$ ). In addition it has to satisfy some additional conditions (see section 2). We consider eq. (2) as an Ansatz for the K-function which transforms these conditions on the K-functions to simple equations for the p-functions. In section 1 we present solutions of these equations.

In this article we propose the p-functions for several local operators. In particular we consider the infinite set of local currents $J_{L}^{ \pm}(x), \quad(L= \pm 1, \pm 3, \ldots)$ belonging to the infinite set of conservation laws which are typical for integrable quantum field theories. For this example the correspondences between the operators, the K-functions and the p-functions are (up to normalization constants)

$$
J_{L}^{ \pm}(x) \leftrightarrow K_{n}^{(L, \pm)}(\underline{\theta}) \leftrightarrow p_{n}^{(L, \pm)}(\underline{\theta}, \underline{l}) \propto \sum_{i=1}^{n} e^{ \pm \theta_{i}} \sum_{i=1}^{n} e^{L\left(\theta_{i}-\frac{i \pi}{2}\left(1-(-1)^{l_{i}} \nu\right)\right)} .
$$

Here the breather p-function is obtained from the corresponding solitonic one [2].

In contrast to this case the breather p-function for exponentials $: e^{i \gamma \varphi}:(x)$ of the field $\varphi$ for generic real $\gamma$ is not related to a solitonic p-function of any local operator (which means that $: e^{i \gamma \varphi}:(x)$ is not local with respect to the soliton field). Now the correspondences are

$$
: e^{i \gamma \varphi}:(x) \leftrightarrow K_{n}^{(q)}(\underline{\theta}) \leftrightarrow p_{n}^{(q)}(\underline{l})=N_{n}^{(q)} \prod_{i=1}^{n} q^{(-1)^{l_{i}}}
$$

\footnotetext{
${ }^{3}$ For the sinh-Gordon model and the case of the exponential field Brazhnikov andLukyanov 23 found by different methods a formula which agrees with our results. Smirnov 17 derived an integral repesentation of sine-Gordon breather form factors (see subsection 6.2 which agrees for some cases with our results (e.g. for the current and the energy momentum tensor) and in the case of the higher currents not (see also footnote 7 ).
} 
where $q=q(\gamma)$ (see section (4). Here and in the following : $\cdots$ : denotes normal ordering with respect to the physical vacuum. This means in particular for the vacuum expectation value $\left\langle 0\left|: \varphi^{N}:(x)\right| 0\right\rangle=0$ and therefore $\langle 0|: \exp i \gamma \varphi$ : $(x)|0\rangle=1$. In section 1 we present arguments to support these correspondences and also determine the normalization constants $N_{n}^{\mathcal{O}}$.

As an application of these results we investigate quantum operator equations. In particular we provide exact expressions for all matrix elements of all powers of the fundamental bose field $\varphi(t, x)$ and its exponential $: \exp i \gamma \varphi:(t, x)$ for arbitrary $\gamma$. We find that the operator $\square^{-1}: \sin \beta \varphi(x)$ : is local. Moreover the quantum sine-Gordon field equation

$$
\square \varphi(x)+m^{2} \frac{\pi \nu}{\sin \pi \nu} \frac{1}{\beta}: \sin \beta \varphi:(x)=0
$$

is fulfilled for all matrix elements. The factor $\frac{\pi \nu}{\sin \pi \nu}$ modifies the classical equation and has to be considered as a quantum correction of the breather mass $m$ as to be compared with the "bare" mass $\sqrt{\alpha}$. Further we find that the trace of the energy momentum tensor $T^{\mu \nu}$ satisfies

$$
T_{\mu}^{\mu}(x)=-2 \frac{\alpha}{\beta^{2}}\left(1-\frac{\beta^{2}}{8 \pi}\right)(: \cos \beta \varphi:(x)-1) .
$$

Again this operator equation is modified by a quantum correction $\left(1-\beta^{2} / 8 \pi\right)$ compared to the classical one.

Also we show that the higher local currents $J_{M}^{\mu}(t, x)$ satisfy $\partial_{\mu} J_{M}^{\mu}(t, x)=0$ and calculate all matrix elements of all higher conserved $Q_{L}=\int d x J_{L}^{0}(t, x)$

$$
Q_{L}\left|p_{1}, \ldots, p_{n}\right\rangle^{i n}=\sum_{i=1}^{n} e^{L \theta_{i}}\left|p_{1}, \ldots, p_{n}\right\rangle^{i n}
$$

In particular for $L= \pm 1$ the currents yield the energy momentum tensor $T^{\mu \nu}=$ $T^{\nu \mu}$ with $\partial_{\mu} T^{\mu \nu}=0$.

The article is organized as follows: In section 2 we recall some formulae of [1, 2] and in particular those for breather form factors, which we need in the following. The properties of the form factors are translated to conditions for the 'K-functions' and finally to simple ones of the 'p-functions'. In section 3 we investigate the asymptotic behavior of bosonic form factors. In section 1 we discuss several explicit examples of local operators as general exponentials of the fundamental bose field, powers of the field, all higher conserved currents, and the energy momentum tensor. Using induction and Liouville's theorem we prove some identities which means that the same operators may be represented in terms of different p-functions. These results are used in section 5 to prove operator field equations as the quantum sine-Gordon field equation. In section 6 we present further types of representations of sine-Gordon breather form factors:

\footnotetext{
${ }^{4}$ In the framework of constructive quantum field theory quantum field equations where considered in 32 33]. For the sine-Gordon model quantum field equations where discussed by Smirnov in 17 and for the sinh-Gordon model in 22] (see also footnote 8).
} 
a determinant formula (see also [17, 19, 20, 21]) and two integral representation. One of them is new and could presumably be generalized to other models with no backward scattering (see also [31]). A proof is delegated to the appendix.

\section{Breather form factors}

Using the bound state fusion method we derived in [2 from a general formula for soliton anti-soliton form factors the pure breather form factor formula which in particular for the case of lowest breathers may be written in the form (11) with $F(\theta)$ being the two particle form factor function. It satisfies Watson's equations

$$
F(\theta)=F(-\theta) S(\theta)=F(2 \pi i-\theta)
$$

with the S-matrix given above. Explicitly it is given by the integral representation 14

$$
F(\theta)=\exp \int_{0}^{\infty} \frac{d t}{t} \frac{\cosh \left(\frac{1}{2}+\nu\right) t-\cosh \frac{1}{2} t}{\cosh \frac{1}{2} t \sinh t} \cosh t\left(1-\frac{\theta}{i \pi}\right)
$$

normalized such that $F(\infty)=1$. In general form factors of one kind of bosonic particles (i.e. with a diagonal S-matrix) satisfy the following properties 14, 16, 11.

Properties of the form factors: The form factor function $\mathcal{O}_{n}(\underline{\theta})$ is meromorphic with respect to all variables $\theta_{1}, \ldots, \theta_{n}$. It satisfies Watson's equations

$$
\mathcal{O}_{n}\left(\ldots, \theta_{i}, \theta_{j}, \ldots\right)=\mathcal{O}_{n}\left(\ldots, \theta_{j}, \theta_{i}, \ldots\right) S\left(\theta_{i j}\right) .
$$

The crossing relation means for the connected part (see e.g. [2]) of the matrix element

$$
\left\langle p_{1}|\mathcal{O}(0)| p_{2}, \ldots, p_{n}\right\rangle_{\text {conn }}^{i n}=\mathcal{O}_{n}\left(\theta_{1}+i \pi, \theta_{2}, \ldots, \theta_{n}\right)=\mathcal{O}_{n}\left(\theta_{2}, \ldots, \theta_{n}, \theta_{1}-i \pi\right)
$$

which implies in particular

$$
\mathcal{O}_{n}\left(\theta_{1}, \theta_{2}, \ldots, \theta_{n},\right)=\mathcal{O}_{n}\left(\theta_{2}, \ldots, \theta_{n}, \theta_{1}-2 \pi i\right)
$$

The function $\mathcal{O}_{n}(\underline{\theta})$ has poles determined by one-particle states in each subchannel. In particular it has the so-called annihilation poles at for example $\theta_{12}=i \pi$ such that the recursion formula $\left.\right|^{5}$ is satisfied

$$
\underset{\theta_{12}=i \pi}{\operatorname{Res}} \mathcal{O}_{n}\left(\theta_{1}, \ldots, \theta_{n}\right)=2 i \mathcal{O}_{n-2}\left(\theta_{3}, \ldots, \theta_{n}\right)\left(\mathbf{1}-S\left(\theta_{2 n}\right) \ldots S\left(\theta_{23}\right)\right) .
$$

Since we are dealing with relativistic quantum field theories Lorentz covariance in the form

$$
\mathcal{O}_{n}\left(\theta_{1}, \ldots, \theta_{n}\right)=e^{-s \mu} \mathcal{O}_{n}\left(\theta_{1}+\mu, \ldots, \theta_{n}+\mu\right)
$$

holds if the local operator transforms as $\mathcal{O} \rightarrow e^{s \mu} \mathcal{O}$ where $s$ is the "spin" of $\mathcal{O}$.

\footnotetext{
${ }^{5}$ This formula has been proposed in 16 , as a generalization of formulae in 14 and it has been proven in [1] using LSZ assumptions.
} 
Conditions on the K-functions: Form factors of one kind of bosonic particles (which means that there is no backward scattering) may be expressed by eq. (11) in terms of the K-functions. Therefore properties of the form factors can be transformed to the following relations:

$$
\begin{aligned}
& K_{n}^{\mathcal{O}}\left(\ldots, \theta_{i}, \theta_{j}, \ldots\right)=K_{n}^{\mathcal{O}}\left(\ldots, \theta_{j}, \theta_{i}, \ldots\right) \\
& K_{n}^{\mathcal{O}}(\underline{\theta})=K_{n}^{\mathcal{O}}\left(\theta_{1}-2 \pi i, \theta_{2}, \ldots, \theta_{n}\right) \\
& \underset{\theta_{12}=i \pi}{\operatorname{Res}} K_{n}^{\mathcal{O}}(\underline{\theta})=\frac{2 i}{F(i \pi)} \prod_{i=3}^{n} \frac{1}{F\left(\theta_{2 i}+i \pi\right) F\left(\theta_{2 i}\right)}\left(1-\prod_{i=3}^{n} S\left(\theta_{2 i}\right)\right) K_{n-2}^{\mathcal{O}}\left(\underline{\theta}^{\prime \prime}(1) 1\right) \\
& K_{n}^{\mathcal{O}}(\underline{\theta})=e^{-s \mu} K_{n}^{\mathcal{O}}\left(\theta_{1}+\mu, \ldots, \theta_{n}+\mu\right)
\end{aligned}
$$

where $\underline{\theta}=\theta_{1}, \ldots, \theta_{n}$ and $\underline{\theta}^{\prime \prime}=\theta_{3}, \ldots, \theta_{n}$.

Equations for the p-functions: Starting with a general integral representation for solitonic form factors and using the bound state fusion method we have shown in 22 that the lowest breather K-functions may be expressed by eq. (2) in terms of breather p-functions which follow from solitonic p-functions. As already mentioned in the introduction in the present article we make the Ansatz that the K-function is of the form (2) and we allow more general breather pfunctions. The Ansatz (2) transforms the conditions on the K-function $K_{n}^{\mathcal{O}}(\underline{\theta})$ to simpler equations for the p-function $p_{n}^{\mathcal{O}}(\underline{\theta}, \underline{l})$. The p-function $p_{n}^{\mathcal{O}}(\underline{\theta}, \underline{l})$ is holomorphic with respect to all variables $\theta_{1}, \ldots, \theta_{n}$, is symmetric with respect to the exchange of the variables $\theta_{i}$ and $l_{i}$ at the same time and is periodic with period $2 \pi i$.

$$
\begin{aligned}
p_{n}^{\mathcal{O}}\left(\ldots, \theta_{i}, \theta_{j}, \ldots, l_{i}, l_{j}, \ldots\right) & =p_{n}^{\mathcal{O}}\left(\ldots, \theta_{j}, \theta_{i}, \ldots, l_{j}, l_{i}, \ldots\right) \\
p_{n}^{\mathcal{O}}(\underline{\theta}, \underline{l}) & =p_{n}^{\mathcal{O}}\left(\theta_{1}-2 \pi i, \theta_{2}, \ldots, \theta_{n}, \underline{l}\right)
\end{aligned}
$$

With the short hand notation $\underline{\theta}^{\prime}=\theta_{2}, \ldots, \theta_{n}, \underline{\theta}^{\prime \prime}=\theta_{3}, \ldots, \theta_{n}$ and $\underline{l}^{\prime \prime}=l_{3}, \ldots, l_{n}$ the recursion relation

$$
p_{n}^{\mathcal{O}}\left(\theta_{2}+i \pi, \underline{\theta}^{\prime}, \underline{l}\right)=g\left(l_{1}, l_{2}\right) p_{n-2}^{\mathcal{O}}\left(\underline{\theta}^{\prime \prime}, \underline{l}^{\prime \prime}\right)+h\left(l_{1}, l_{2}\right)
$$

holds where $g(0,1)=g(1,0)=2 /(F(i \pi) \sin \pi \nu)$ and $h\left(l_{1}, l_{2}\right)$ is independent of $\underline{l}^{\prime \prime}$. Lorentz covariance reads as

$$
p_{n}^{\mathcal{O}}\left(\theta_{1}+\mu, \ldots, \theta_{n}+\mu, \underline{l}\right)=e^{s \mu} p_{n}^{\mathcal{O}}\left(\theta_{1}, \ldots, \theta_{n}, \underline{l}\right) .
$$

We now show that these conditions of the p-function are sufficient to guarantee the properties of the form factors.

Theorem 1 If the p-function $p_{n}^{\mathcal{O}}(\underline{\theta}, \underline{l})$ satisfies the conditions (13] 10$)$, the $K$ function $K_{n}^{\mathcal{O}}(\underline{\theta})$ satisfies the conditions $(9-19)$ and therefore the form factor function $\mathcal{O}_{n}(\underline{\theta})$ satisfies the properties (5) 8 . 
Proof. Except for (11) all claims are obvious. Taking the residue of (21) and inserting (11) we obtain (with $a=i \sin \pi \nu$ )

$$
\begin{aligned}
& \underset{\theta_{12}=i \pi}{\operatorname{Res}} K_{n}(\underline{\theta})=-a \sum_{l_{3}=0}^{1} \cdots \sum_{l_{r}=0}^{1}(-1)^{l_{3}+\cdots+l_{n}} \prod_{3=i<j}^{n}\left(1+\frac{l_{i}-l_{j}}{\sinh \theta_{i j}} a\right) \\
& \times \sum_{l_{1} \neq l_{2}}(-1)^{l_{1}+l_{2}}\left(l_{1}-l_{2}\right) \prod_{i=3}^{n}\left(\left(1+\frac{l_{1}-l_{i}}{\sinh \left(\theta_{2 i}+i \pi\right)} a\right)\left(1+\frac{l_{2}-l_{i}}{\sinh \theta_{2 i}} a\right)\right) \\
& \times\left(g\left(l_{1}, l_{2}\right) p_{n-2}^{\mathcal{O}}\left(\underline{\theta}^{\prime \prime}, \underline{l}^{\prime \prime}\right)+h\left(l_{1}, l_{2}\right)\right) \\
& =\frac{2 i}{F(i \pi)} K_{n-2}\left(\underline{\theta}^{\prime \prime}\right)\left(\prod_{i=3}^{n}\left(1+\frac{a}{\sinh \theta_{2 i}}\right)-\prod_{i=3}^{n}\left(1-\frac{a}{\sinh \theta_{2 i}}\right)\right)+h \text {-term. }
\end{aligned}
$$

We have used the identity

$$
\begin{array}{r}
\sum_{l_{1} \neq l_{2}}(-1)^{l_{1}+l_{2}}\left(l_{1}-l_{2}\right) \prod_{i=3}^{n}\left(\left(1+\frac{l_{1}-l_{i}}{\sinh \left(\theta_{2 i}+i \pi\right)} a\right)\left(1+\frac{l_{2}-l_{i}}{\sinh \theta_{2 i}} a\right)\right) g\left(l_{1}, l_{2}\right) \\
=g(0,1) \prod_{i=3}^{n}\left(1+\frac{a}{\sinh \theta_{2 i}}\right)-g(1,0) \prod_{i=3}^{n}\left(1-\frac{a}{\sinh \theta_{2 i}}\right)
\end{array}
$$

valid for all $l_{i}(i \geq 3)$. The same relation is valid when we replace $g$ by $h$. The equation (11) now follows from $g(0,1)=g(1,0)=2 /(F(i \pi) \sin \pi \nu)$ and

$$
F(\theta+i \pi) F(\theta)=1 /\left(1-\frac{i \sin \pi \nu}{\sinh \theta}\right)
$$

(which is easily obtained from the integral representation (4)), provided that the $h$-term vanishes. This is a consequence of the following lemma. Note that the $h$-term is proportional to a $K_{n-2}\left(\underline{\theta}^{\prime \prime}\right)$ given by the formula (2) with a p-function independent of the $\underline{l}^{\prime \prime}$.

Lemma 2 If the 'p-function' in (园) does not depend on $l_{1}, \ldots, l_{n}$ then the corresponding $K$-function vanishes.

Proof. The proof is easy and obtained by using induction and Liouville's theorem: We easily obtain $K_{1}(\underline{\theta})=K_{2}(\underline{\theta})=0$. As induction assumptions we take $K_{n-2}\left(\underline{\theta}^{\prime \prime}\right)=0$. The function $K_{n}(\underline{\theta})$ is a meromorphic functions in terms of the $x_{i}=e^{\theta_{i}}$ with at most simple poles at $x_{i}= \pm x_{j} \operatorname{since} \sinh \theta_{i j}=$ $\left(x_{i}+x_{j}\right)\left(x_{i}-x_{j}\right) /\left(2 x_{i} x_{j}\right)$. The residues of the poles at $x_{i}=x_{j}$ vanish because of the symmetry. Furthermore the residues at $x_{i}=-x_{j}$ are proportional to $K_{n-2}\left(\underline{\theta}^{\prime \prime}\right)$ because similarly to the proof of theorem 1 we have

$$
\underset{\theta_{12}=i \pi}{\operatorname{Res}} K_{n}(\underline{\theta})=a K_{n-2}\left(\underline{\theta}^{\prime \prime}\right)\left(\prod_{i=3}^{n}\left(1+\frac{a}{\sinh \theta_{2 i}}\right)-\prod_{i=3}^{n}\left(1-\frac{a}{\sinh \theta_{2 i}}\right)\right)
$$


Therefore the function $K_{n}(\underline{\theta})$ is holomorphic everywhere. Furthermore for $x_{1} \rightarrow$ $\infty$ we have the asymptotic behavior

$$
\begin{array}{r}
K_{n}(\underline{\theta})=\sum_{l_{2}=0}^{1} \cdots \sum_{l_{n}=0}^{1}(-1)^{l_{2}+\cdots+l_{n}} \prod_{2 \leq i<j \leq n}\left(1+\left(l_{i}-l_{j}\right) \frac{i \sin \pi \nu}{\sinh \theta_{i j}}\right) \\
\times \sum_{l_{1}=0}^{1}(-1)^{l_{1}} \prod_{j=2}^{n}\left(1+\left(l_{1}-l_{j}\right) \frac{i \sin \pi \nu}{\sinh \theta_{1 j}}\right) \rightarrow 0
\end{array}
$$

Therefore $K_{n}(\underline{\theta})$ vanishes identically by Liouville's theorem.

\section{Asymptotic behavior of bosonic form factors}

In this section we derive the asymptotic behavior of bosonic form factors by mean of general techniques of renormalized local quantum field theory. In particular we use perturbation theory in term of Feynman graphs. As the simplest example we investigate first the asymptotic behavior for $p_{1} \rightarrow \infty$ or $\theta_{12} \rightarrow \infty$ of

$$
\begin{aligned}
\left\langle 0\left|: \varphi^{2}:\right| p_{1}, p_{2}\right\rangle^{\text {in }} & =2\left\langle 0|\varphi(0)| p_{1}\right\rangle\left\langle 0|\varphi(0)| p_{2}\right\rangle+o(1) \\
& =2 Z^{\varphi}+o(1)
\end{aligned}
$$

where : $\cdots$ : means normal ordering with respect to the physical vacuum. This may be seen in perturbation theory as follows: Feynman graph expansion in lowest order means

$$
\begin{aligned}
\left\langle 0\left|: \varphi^{2}:\right| p_{1}, p_{2}\right\rangle^{i n}=2( \\
=2\left(1+i \alpha \beta^{2} \frac{1}{2} \int \frac{d^{2} k}{(2 \pi)^{2}} \frac{i}{k^{2}-m_{1}^{2}} \frac{i}{(p-k)^{2}-m_{1}^{2}}\right)+O\left(\beta^{4}\right) \\
=2+\frac{\beta^{2}}{4 \pi} \frac{i \pi-\theta_{12}}{\sinh \theta_{12}}+O\left(\beta^{4}\right)
\end{aligned}
$$

The second graph is of order $O\left(\ln p_{1} / p_{1}\right)$ for $p_{1} \rightarrow \infty$. This is typical also for all orders in perturbation theory:

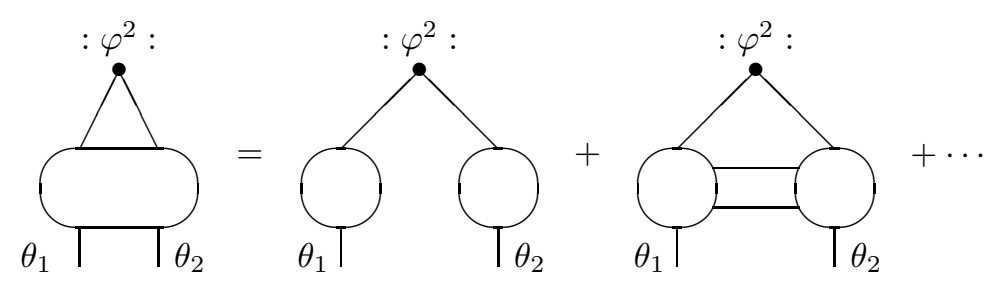


Weinberg's power counting theorem says that the second term and also all higher terms where more lines connect the two bubbles are again at least of order $O\left(\ln p_{1} / p_{1}\right)$ for $p_{1} \rightarrow \infty$.

The wave function renormalization constant $Z^{\varphi}$ is defined as usual by the two-point function of the (unrenormalized) field

$$
\begin{aligned}
\int\langle 0|T \varphi(x) \varphi(0)| 0\rangle e^{i p x} d^{2} x & =-+\bigcirc+\bigcirc \\
& =\frac{i}{p^{2}-\alpha-\Pi\left(p^{2}\right)}=\frac{i Z^{\varphi}}{p^{2}-m^{2}-\Pi_{r e n}\left(p^{2}\right)}
\end{aligned}
$$

where $\Pi(p)$ is the self energy, which means that it is given by the sum of all amputated one-particle-irreducible graphs

$$
-i \Pi\left(p^{2}\right)=-\square
$$

The physical breather mass $m$, the wave function renormalization constant $Z^{\varphi}$ and the renormalized breather self energy are given by

$$
\begin{aligned}
m^{2} & =\alpha+\Pi\left(m^{2}\right) \\
\frac{1}{Z^{\varphi}} & =1-\Pi^{\prime}\left(m^{2}\right) \\
\Pi_{r e n}\left(p^{2}\right) & =Z^{\varphi}\left(\Pi\left(p^{2}\right)-\Pi\left(m^{2}\right)-\left(p^{2}-m^{2}\right) \Pi^{\prime}\left(m^{2}\right)\right) .
\end{aligned}
$$

Since the sine-Gordon model is a 'super renormalizable quantum field theory' both renormalization constants $\Pi\left(m^{2}\right)$ and $\Pi^{\prime}\left(m^{2}\right)$ become finite after taking normal ordering in the interaction Lagrangian.. They can be calculated exactly. The wave function renormalization constant was obtained in [14]

$$
Z^{\varphi}=(1+\nu) \frac{\frac{\pi}{2} \nu}{\sin \frac{\pi}{2} \nu} \exp \left(-\frac{1}{\pi} \int_{0}^{\pi \nu} \frac{t}{\sin t} d t\right)
$$

and the relation of the unrenormalized and the physical mass is calculated in the present article (see section 5)

$$
\alpha=m^{2} \frac{\pi \nu}{\sin \pi \nu} .
$$

Both relations have been checked in perturbation theory.

Remark 3 Usually in renormalized quantum field theory (in particular when $Z$ is infinite) one would introduce the renormalized field with

$$
\left\langle 0\left|\varphi_{\text {ren }}(0)\right| p\right\rangle=1
$$


by $\varphi(x)=\sqrt{Z^{\varphi}} \varphi_{\text {ren }}(x)$. Since Coleman's article 4 however, the convention for the sine-Gordon model is to use the unrenormalized field $\varphi(x)$ which is related to the massive Thirring model current by

$$
j^{\mu}=-\frac{\beta}{2 \pi} \epsilon^{\mu \nu} \partial_{\nu} \varphi
$$

Therefore we have the normalization

$$
\langle 0|\varphi(0)| p\rangle=\sqrt{Z^{\varphi}}
$$

The wave function renormalization constant $Z^{\varphi}$ is plotted as a function of $\nu$ in figure 1 (a) for negative values of $\nu$ which correspond to the sinh-Gordon model and in (b) for $0 \leq \nu \leq 1$ which correspond to the sine-Gordon model for $0 \leq \beta^{2} \leq 4 \pi$. Note that in (a) the function is symmetric with respect to the self-dual point $\nu=1 / 2$ of the sinh-Gordon model and that in (b) $Z^{\varphi}=1$ for the free breather point $\nu=0$ and $Z^{\varphi}=0$ for the free fermi point $\nu=1$ where the breather disappears from the particle spectrum.

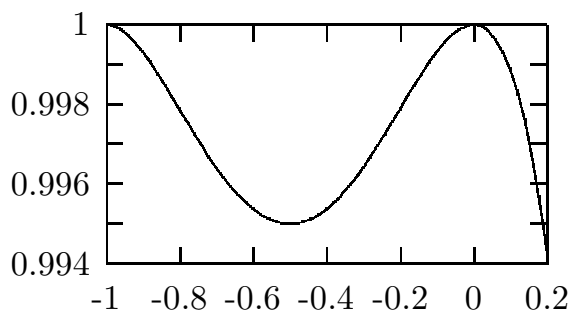

(a)

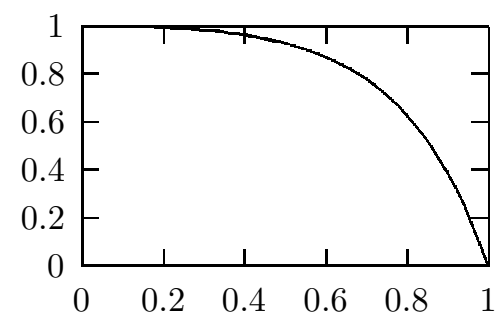

(b)

Figure 1: The wave function renormalization constant $Z^{\varphi}$ given by eq. (18) as a function of $\nu$.

As a generalization we now consider general n-particle form factors of an normal ordered arbitrary power of the field $\mathcal{O}=: \varphi^{N}$ : and let $m$ of the momenta tend to infinity. If $\underline{\theta}(\lambda)=\left(\theta_{1}+\lambda, \ldots, \theta_{m}+\lambda, \theta_{m+1}, \ldots, \theta_{n}\right), \underline{\theta}^{\prime}=\left(\theta_{1}, \ldots, \theta_{m}\right)$ and $\underline{\theta}^{\prime \prime}=\left(\theta_{m+1}, \ldots, \theta_{n}\right)$ Weinberg's power counting theorem for bosonic Feyn- 
man graphs says that for $\operatorname{Re} \lambda \rightarrow \infty$

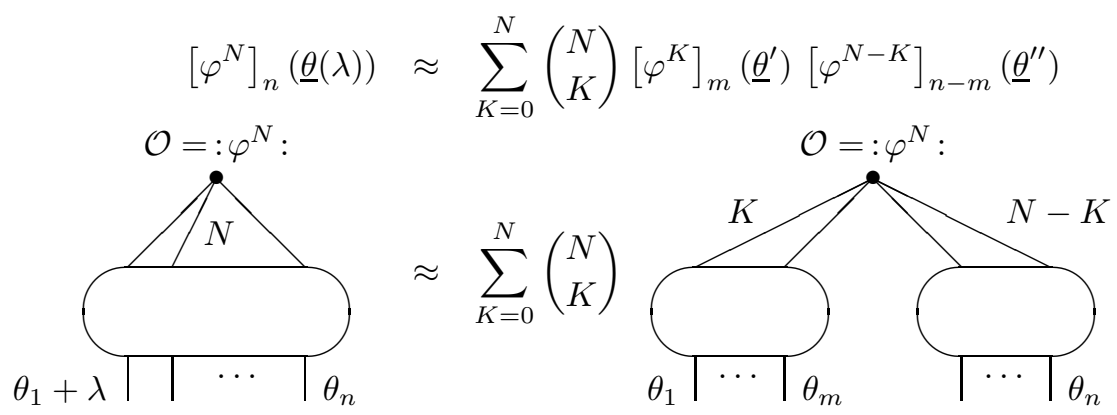

with the notation $\left[\varphi^{N}\right]_{n}(\underline{\theta})=\left\langle 0\left|: \varphi^{N}:(0)\right| p_{1}, \ldots, p_{n}\right\rangle^{i n}$. For the special case of a local operator which is an exponential of the fundamental bose field $\mathcal{O}=: e^{i \gamma \varphi}:($ for some $\gamma$ ) we therefore have

$$
\left[e^{i \gamma \varphi}\right]_{n}(\underline{\theta}(\lambda))=\left[e^{i \gamma \varphi}\right]_{m}\left(\underline{\theta}^{\prime}\right)\left[e^{i \gamma \varphi}\right]_{n-m}\left(\underline{\theta}^{\prime \prime}\right)+O\left(e^{-\lambda}\right) .
$$

This gives in particular for $m=1$ and $\operatorname{Re} \theta_{1} \rightarrow \infty$

$$
\left[e^{i \gamma \varphi}\right]_{n}\left(\theta_{1}, \theta_{2}, \ldots, \theta_{n}\right)=\left[e^{i \gamma \varphi}\right]_{1}\left(\theta_{1}\right)\left[e^{i \gamma \varphi}\right]_{n-1}\left(\theta_{2}, \ldots, \theta_{n}\right)+O\left(e^{-\theta_{1}}\right) .
$$

\section{Examples of operators}

In this section we present some examples of p-functions which satisfy the conditions of section 2 in particular (13) - (16) and propose the correspondence of local operators, K-functions and p-functions due to eqs. (11) and (2) for these examples

$$
\mathcal{O} \leftrightarrow K_{n}^{\mathcal{O}}(\underline{\theta}) \leftrightarrow p_{n}^{\mathcal{O}}(\underline{\theta}, \underline{l})
$$

\subsection{Classical local operators}

The classical sine-Gordon Lagrangian is

$$
\mathcal{L}^{S G}=\frac{1}{2} \partial_{\mu} \varphi \partial^{\mu} \varphi+\frac{\alpha}{\beta^{2}}(\cos \beta \varphi-1)
$$

We consider the following classical local operators:

1. $\exp (i \gamma \varphi(x))$ for arbitrary real $\gamma$,

2. $\varphi^{N}(x)$

3. higher conserved currents for $(L=1,3,5 \ldots)$

$$
J_{L}^{\rho}=\left\{\begin{array}{c}
J_{L}^{+}=\partial^{+} \varphi\left(\partial^{+}\right)^{L} \varphi+O\left(\varphi^{4}\right) \\
J_{L}^{-}=\left(\left(\partial^{+}\right)^{L-1} \varphi+O\left(\varphi^{2}\right)\right) \sin \varphi
\end{array} .\right.
$$


A second set of conserved currents is obtained by replacing $\partial^{+} \rightarrow \partial^{-}$. The higher charges are of the form

$$
Q_{L}=\int d x\left(\partial^{0} \varphi \partial^{+L} \varphi+O\left(\varphi^{4}\right)\right), \quad L=1,3,5, \ldots
$$

and the charges for even $L$ vanish.

4. $T^{\mu \nu}(x)=\partial^{\mu} \varphi \partial^{\nu} \varphi-g^{\mu \nu} \mathcal{L}^{S G}$ the energy momentum tensor or in terms of light cone coordinates $\left(\partial^{ \pm}=\partial^{0} \pm \partial^{1}\right.$ etc. $)$

$$
\begin{array}{r}
T^{ \pm \pm}=T^{00} \pm T^{01} \pm T^{10}+T^{11}=\partial^{+} \varphi \partial^{+} \varphi=\partial^{-} \varphi \partial^{-} \varphi \\
T^{+-}=T^{00}-T^{01}+T^{10}-T^{11}=-2 \frac{\alpha}{\beta^{2}}(\cos \beta \varphi-1)=T^{-+}
\end{array}
$$

5. $\exp (i \beta \varphi(x))$ for the particular value $\gamma=\beta$

\subsection{The normalization of form factors}

The normalization constants are obtained in the various cases by the following observations:

a) The normalization a field annihilating a one-particle state is given by the vacuum one-particle matrix element, in particular for the fundamental bose field one has

$$
\langle 0|\varphi(0)| p\rangle=\sqrt{Z^{\varphi}} .
$$

$Z^{\varphi}$ is the finite wave function renormalization constant (18) which has been calculated in [14.

b) If an observable like a charge $Q=\int d x \mathcal{O}(x)$ belongs to a local operator we use the relation

$$
\left\langle p^{\prime}|Q| p\right\rangle=q\left\langle p^{\prime} \mid p\right\rangle .
$$

This will be applied for example to the higher conserved charges.

c) We use Weinberg's power counting theorem for bosonic Feynman graphs. As discussed in section 3 this yields in particular the asymptotic behavior for the exponentials of the boson field $\mathcal{O}=: e^{i \gamma \varphi}$ :

$$
\mathcal{O}_{n}\left(\theta_{1}, \theta_{2}, \ldots\right)=\mathcal{O}_{1}\left(\theta_{1}\right) \mathcal{O}_{n-1}\left(\theta_{2}, \ldots\right)+O\left(e^{-\operatorname{Re} \theta_{1}}\right)
$$

as $\operatorname{Re} \theta_{1} \rightarrow \infty$ in any order of perturbation theory. This behavior is also assumed to hold for the exact form factors. Applying this formula iteratively we obtain from (2) relations ${ }^{6}$ for the normalization constants of the operators : $e^{i \gamma \varphi}$ :

\footnotetext{
${ }^{6}$ This type of arguments has been also used in 14, 20, 21, 22.
} 
d) The recursion relation (15) relates $N_{n+2}$ and $N_{n}$. For all p-functions discussed below this means

$$
N_{n+2}=N_{n} \frac{2}{\sin \pi \nu F(i \pi)} \quad(n \geq 1) .
$$

where $F(i \pi)$ is related to the wave function renormalization constant by

$$
\frac{1}{F(i \pi)}=\frac{\beta^{2}}{8 \pi \nu} \frac{\sin \pi \nu}{\pi \nu} Z^{\varphi}
$$

see [2] and eq. (18).

\subsection{Local operators and their p-functions}

For all cases to be discussed in the following, the conditions (13 16) are again obvious except that of the recursion relation (15) which will be discussed in detail. For later convenience we also list for some cases the explicit expressions of $K_{n}(\underline{\theta})$ for $n=1,2$ and the asymptotic behavior of $K_{n}(\underline{\theta})$ for $\operatorname{Re} \theta_{1} \rightarrow \infty$ which is easily obtained analogously to the calculation (17) in the proof of lemma 2 . For convenience we will use the notation $K_{n}=N_{n} K_{n}$ in the following.

\subsubsection{Exponentials of the breather field}

We propose the correspondence

$$
e^{i \gamma \varphi} \leftrightarrow N_{n}^{(q)} \tilde{K}_{n}^{(q)}(\underline{\theta}) \leftrightarrow p_{n}^{(q)}(\underline{l})=N_{n}^{(q)} \prod_{i=1}^{n} q^{(-1)^{l_{i}}}
$$

with $q=q(\gamma)$ (and $q(0)=1$ ) to be determined below. For low particle numbers one easily calculates the K-functions

$$
\begin{aligned}
\tilde{K}_{1}^{(q)}(\theta) & =(q-1 / q) \\
\tilde{K}_{2}^{(q)}(\underline{\theta}) & =(q-1 / q)^{2}
\end{aligned}
$$

and the asymptotic behavior

$$
\tilde{K}_{n}^{(q)}(\underline{\theta}) \approx \tilde{K}_{1}^{(q)}\left(\theta_{1}\right) \tilde{K}_{n-1}^{(q)}\left(\underline{\theta}^{\prime}\right) .
$$

The last formula is obtained similarly to the proof of lemma 2. The proposal that the p-function $p_{n}^{(q)}(\underline{l})$ correspond to an exponential of a bosonic field is supported by the following observation. The asymptotic behavior is consistent with that of the form factors of exponentials of bosonic fields $(20)$ as discussed in section 3. Indeed it reads in terms of the K-functions as $K_{n}^{(q)}(\underline{\theta}) \approx K_{1}^{(q)}\left(\theta_{1}\right) K_{n-1}^{(q)}\left(\underline{\theta}^{\prime}\right)$ (since $F(\infty)=1$ ) provided the normalization constants satisfy

$$
N_{n}^{(q)}=N_{1}^{(q)} N_{n-1}^{(q)} \quad \Rightarrow \quad N_{n}^{(q)}=\left(N_{1}^{(q)}\right)^{n} .
$$


This is what we discussed above under point c) to determine the normalization constants. Point $d$ ) in the present case has the following meaning. The recursion condition (15) is satisfied since in this case we have $g\left(l_{1}, l_{2}\right)=$

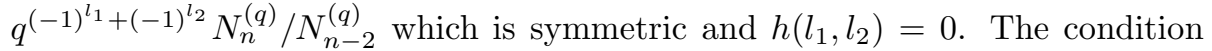
(15) with $g(0,1)=g(1,0)=2 /(F(i \pi) \sin \pi \nu)$ implies the recursion relation for the normalization constants (22) which finally yields

$$
\begin{aligned}
N_{1}^{(q)} & =\sqrt{\frac{2}{F(i \pi) \sin \pi \nu}}=\sqrt{Z^{\varphi}} \frac{\beta}{2 \pi \nu} \\
N_{n}^{(q)} & =\left(\sqrt{Z^{\varphi}} \frac{\beta}{2 \pi \nu}\right)^{n}
\end{aligned}
$$

where $Z^{\varphi}$ is the breather wave function renormalization constant (18). The relation of $F(i \pi)$ with $Z^{\varphi}$ is obtained by elementary manipulations of the integral representation (舟) and (18). Recall that normal ordering implies $N_{0}^{(q)}=1$.

\subsubsection{Powers of the breather field}

Motivated by the expansion of (23) with respect to $\ln q$ we propose the correspondence

$$
\varphi^{N} \leftrightarrow N_{n}^{(N)} \tilde{K}_{n}^{(N)}(\underline{\theta}) \leftrightarrow p_{n}^{(N)}(\underline{l})=N_{n}^{(N)}\left(\sum_{i=1}^{n}(-1)^{l_{i}}\right)^{N}
$$

Again one easily calculates (with $\tilde{K}_{n}^{(N)}=K_{n}^{(N)} / N_{n}^{(N)}$ ) the low particle number K-functions

$$
\begin{aligned}
\tilde{K}_{1}^{(N)}(\theta) & =2 \\
\tilde{K}_{2}^{(N)}(\underline{\theta}) & =2^{N+1} \\
\tilde{K}_{3}^{(N)}(\underline{\theta}) & =2\left(3^{N}-3\right)-\sin ^{2} \pi \nu \prod_{i<j} \frac{1}{\cosh \frac{1}{2} \theta_{i j}}
\end{aligned}
$$

and the asymptotic behavior

$$
\tilde{K}_{n}^{(N)}(\underline{\theta}) \approx \sum_{K=1}^{N}\left(\begin{array}{l}
N \\
K
\end{array}\right) \tilde{K}_{1}^{(K)}\left(\theta_{1}\right) \tilde{K}_{n-1}^{(N-K)}\left(\underline{\theta}^{\prime}\right)
$$

where $K_{n}^{(N)}$ is only nonvanishing for $N-n=$ even. This asymptotic behavior agrees with (19) which follows from Weinberg's power counting argument and therefore justifies the correspondence $(26)$. The normalization condition $\langle 0|\varphi(0)| p\rangle=\sqrt{Z^{\varphi}}($ see $(21)$ yields

$$
N_{1}^{(1)}=\frac{1}{2} \sqrt{Z^{\varphi}} .
$$

The other normalization constants and also the function $q(\gamma)$ is now obtained as follows. Comparing the correspondences (23) and (26) we conclude

$$
N_{n}^{(N)}=N_{n}^{(q)}\left(\frac{\ln q}{i \gamma}\right)^{N} .
$$


This implies for $N=n=1$ together with (25)

$$
q=\exp \left(i \gamma \frac{N_{1}^{(1)}}{N_{1}^{(q)}}\right)=\exp \left(i \frac{\pi \nu}{\beta} \gamma\right) .
$$

and finally the normalization constants

$$
N_{n}^{(N)}=\left(\frac{1}{2} \sqrt{Z^{\varphi}}\right)^{n}\left(\frac{\pi \nu}{\beta}\right)^{N-n} .
$$

We compare these general results with known special cases [14]. In particular for $n=N=2$ we have

$$
\begin{aligned}
\left\langle 0\left|: \varphi^{2}:(0)\right| p_{1}, p_{2}\right\rangle^{i n} & =K_{2}^{(2)}\left(\theta_{12}\right) F\left(\theta_{12}\right)=8 N_{2}^{(2)} F\left(\theta_{12}\right) \\
& =2 Z^{\varphi} F\left(\theta_{12}\right)
\end{aligned}
$$

which agrees with formulae (4.4-4.6) of [14. Further for $n=3$ and $N=1$ we have

$$
\begin{aligned}
\left\langle 0|\varphi(0)| p_{1}, p_{2}, p_{3}\right\rangle^{i n} & =K_{3}^{(1)}(\underline{\theta}) \prod_{i<j} F\left(\theta_{i j}\right) \\
& =-\left(Z^{\varphi}\right)^{3 / 2} \frac{1}{8}\left(\beta \frac{\sin \pi \nu}{\pi \nu}\right)^{2} \prod_{i<j} \frac{F\left(\theta_{i j}\right)}{\cosh \frac{1}{2} \theta_{i j}}
\end{aligned}
$$

which again agrees with formulae (4.9-4.12) of [14].

\subsubsection{Higher conserved currents}

In the following we present new results concerning the higher conservation laws which are typical for integrable quantum field theories?. We propose the correspondence

$$
J_{L}^{ \pm} \leftrightarrow p_{n}^{(L, \pm)}(\underline{\theta}, \underline{l})= \pm N_{n}^{\left(J_{L}\right)} \sum_{i=1}^{n} e^{ \pm \theta_{i}} \sum_{i=1}^{n} e^{L\left(\theta_{i}-\frac{i \pi}{2}\left(1-(-1)^{l_{i}} \nu\right)\right)}
$$

for $n=$ even and zero for $n=\operatorname{odd}(L= \pm 1, \pm 3, \ldots)$. Again one easily calculates the 2 particle K-function

$$
\tilde{K}_{2}^{(L, \pm)}(\underline{\theta})=-2(-i)^{L} \sin \frac{1}{2} L \pi \nu \frac{\sin \pi \nu}{\sinh \theta_{12}}\left(e^{ \pm \theta_{1}}+e^{ \pm \theta_{2}}\right)\left(e^{L \theta_{1}}-e^{L \theta_{2}}\right)
$$

\footnotetext{
${ }^{7}$ In 17 form factors of higher currents in the sine-Gordon model were proposed, however the charges of these currents vanish. The densities where proposed to be of the form $\left(\partial^{+}\right)^{L} \partial^{1} A(x)$ where the operator $A$ is related to the energy momentum tensor, in particular $\partial^{0} \partial^{1} A=T^{10}$ and $\partial^{1} \partial^{1} A=T^{00}$. For $L>1$ obviously $\int d x\left(\partial^{+}\right)^{L} \partial^{1} A(x)=$ $\int d x\left(\partial^{+}\right)^{L-2} \partial^{1}\left(T^{10}+T^{00}+T^{01}+T^{00}\right)=0$ where the conversation laws $\partial^{0} T^{\mu 0}=\partial^{1} T^{\mu 1}$ have been used.
} 
The recursion condition (15) is satisfied since $g\left(l_{1}, l_{2}\right)=N_{n}^{\left(J_{L}\right)} / N_{n-2}^{\left(J_{L}\right)}$ is symmetric and $h\left(l_{1}, l_{2}\right)=\sum_{i=3}^{n} e^{ \pm \theta_{i}} \sum_{i=1}^{2} e^{L\left(\theta_{i}-\frac{i \pi}{2}\left(1-(-1)^{l_{i}} \nu\right)\right)}$ is independent of $l_{i},(i>2)$. Again we have the recursion relation for the normalization constants (22). The two particle normalization we calculated by means of b) with the charges

$$
\begin{aligned}
\left\langle p^{\prime}\left|Q_{L}\right| p\right\rangle & =\int_{-\infty}^{\infty} d x\left\langle p^{\prime}\left|\frac{1}{2}\left(J_{L}^{+}(x)+J_{L}^{-}(x)\right)\right| p\right\rangle \\
& =\int_{-\infty}^{\infty} d x e^{i\left(p-p^{\prime}\right) x} \frac{1}{2}\left(K_{2}^{(L,+)}+K_{2}^{(L,-)}\right)\left(\theta^{\prime}+i \pi, \theta\right) F\left(\theta^{\prime}+i \pi-\theta\right) \\
& =2 \pi \delta\left(p-p^{\prime}\right) \frac{1}{2}\left(K_{2}^{(L,+)}+K_{2}^{(L,-)}\right)(\theta+i \pi, \theta) F(i \pi) \\
& =\left\langle p^{\prime} \mid p\right\rangle e^{L \theta} \quad \text { if } L \text { odd. }
\end{aligned}
$$

Using (28) we obtain

$$
\begin{aligned}
& \frac{1}{2}\left(K_{2}^{(L,+)}+K_{2}^{(L,-)}\right)(\theta+i \pi, \theta) \\
& =-N_{n}^{\left(J_{L}\right)} 2(-i)^{L} \sin \frac{1}{2} L \pi \nu \sin \pi \nu \cosh \theta e^{L \theta}\left(e^{L i \pi}-1\right) \\
& =2 m \cosh \theta e^{L \theta} / F(i \pi)
\end{aligned}
$$

for $L$ odd. For even $L$ the charges vanish as in the classical case. With the relation of the normalization constants (22) we finally obtain

$$
N_{n}^{\left(J_{L}\right)}=\frac{m i^{L}}{4 \sin \frac{1}{2} L \pi \nu}\left(\sqrt{Z^{\varphi}} \frac{\beta}{2 \pi \nu}\right)^{n} .
$$

Next we derive all eigenvalues of the higher charges (3). We show that for $n^{\prime}+$ $n>2$ the connected part of the matrix element ${ }^{\text {out }}\left\langle p_{1}^{\prime}, \ldots, p_{n^{\prime}}^{\prime}\left|Q_{L}\right| p_{1}, \ldots, p_{n}\right\rangle^{\text {in }}$ vanishes. The analytic continuation $\mathcal{O}_{n^{\prime}+n}\left(\underline{\theta^{\prime}}+i \pi, \underline{\theta}\right)$ yields this connected part. From the correspondence of operators and p-functions

$$
\begin{aligned}
Q_{L}=\int d x J_{L}^{0}(x) & \leftrightarrow 2 \pi \delta\left(P^{\prime}-P\right) N_{n}^{\left(J_{L}\right)} m\left(-\sum_{i=1}^{n^{\prime}} \sinh \theta_{i}^{\prime}+\sum_{i=1}^{n} \sinh \theta_{i}\right) \\
\times & \left(\sum_{i=1}^{n^{\prime}} e^{L\left(\theta_{i}^{\prime}+i \pi-\frac{i \pi}{2}\left(1-(-1)^{l_{i}} \nu\right)\right)}+\sum_{i=1}^{n} e^{L\left(\theta_{i}-\frac{i \pi}{2}\left(1-(-1)^{l_{i}} \nu\right)\right)}\right)
\end{aligned}
$$

the claim follows since for $n^{\prime}+n>2$ there are no poles which may cancel the zero at $P^{\prime}=P$ where $P^{(\prime)}=\sum p_{i}^{(\prime)}$. Therefore contributions to matrix element come from disconnected parts which contain (analytic continued) two-particle 
form factors:

$$
\begin{aligned}
& { }^{\text {out }}\left\langle p_{1}^{\prime}, \ldots, p_{n^{\prime}}^{\prime}\left|Q_{L}\right| p_{1}, \ldots, p_{n}\right\rangle^{\text {in }} \\
& =\sum_{i, j}{ }^{\text {out }}\left\langle p_{1}^{\prime}, \ldots, \hat{p}_{i}^{\prime}, \ldots, p_{n^{\prime}}^{\prime} \mid p_{1}, \ldots, \hat{p}_{j}, \ldots, p_{n}\right\rangle^{\text {in }}\left\langle p_{i}^{\prime}\left|Q_{L}\right| p_{j}\right\rangle \\
& ={ }^{\text {out }}\left\langle p_{1}^{\prime}, \ldots, p_{n^{\prime}}^{\prime} \mid p_{1}, \ldots, p_{n}\right\rangle^{\text {in }} \sum_{i=1}^{n} e^{\theta_{i} L}
\end{aligned}
$$

where $\hat{p}_{j}$ means that this particles is missing in the state.

From the higher currents for $L= \pm 1$ we get the light cone components of the energy momentum tensor $T^{\rho \sigma} \propto J_{\sigma}^{\rho}$ with $\rho, \sigma= \pm$ (see also [22]).

\subsubsection{The energy momentum tensor}

We propose the correspondence

$$
T^{\rho \sigma} \leftrightarrow p_{n}^{\rho \sigma}(\underline{\theta}, \underline{l})=\rho N_{n}^{(T)} \sum_{i=1}^{n} e^{\rho \theta_{i}} \sum_{i=1}^{n} e^{\sigma\left(\theta_{i}-\frac{i \pi}{2}\left(1-(-1)^{l_{i}} \nu\right)\right)}
$$

for $n$ even and $p_{n}^{\rho \sigma}=0$ for $n$ odd. The normalization is again determined by c) namely

$$
\left\langle p^{\prime}\left|P^{\nu}\right| p\right\rangle=\left\langle p^{\prime}\left|\int d x T^{0 \nu}(x)\right| p\right\rangle=\left\langle p^{\prime} \mid p\right\rangle p^{\nu}
$$

which in analogy to (29) gives

$$
N_{n}^{(T)}=\frac{i m^{2}}{4 \sin \frac{1}{2} \pi \nu}\left(\sqrt{Z^{\varphi}} \frac{\beta}{2 \pi \nu}\right)^{n} .
$$

Note that at the first sight the energy momentum tensor does not seem to be symmetric. However, it is due to an identity proven in the next section (see theorem 5). The conservation law follows as above for the higher currents and also the eigenvalue equation of the energy momentum operator with the result

$$
P^{\nu}\left|p_{1}, \ldots, p_{n}\right\rangle^{i n}=\sum_{i=1}^{n} p_{i}^{\nu}\left|p_{1}, \ldots, p_{n}\right\rangle^{i n} .
$$

\subsubsection{Special exponentials of the breather field}

For the special cases of the exponential of the field $\gamma= \pm \beta$ we propose the alternative correspondence to (23)

$$
e^{ \pm i \beta \varphi} \leftrightarrow N_{n}^{ \pm} \tilde{K}_{n}^{ \pm}(\underline{\theta}) \leftrightarrow p_{n}^{ \pm}(\underline{\theta}, \underline{l})=N_{n}^{ \pm} \sum_{i=1}^{n} e^{\mp \theta_{i}} \sum_{i=1}^{n} e^{ \pm\left(\theta_{i}-\frac{i \pi}{2}\left(1-(-1)^{l_{i}} \nu\right)\right)} .
$$

Again one easily calculates for low particle number the K-functions

$$
\begin{aligned}
& \tilde{K}_{1}^{ \pm}(\theta)=2 \sin \frac{1}{2} \pi \nu \\
& \tilde{K}_{2}^{ \pm}(\underline{\theta})= \pm 4 i \sin \frac{1}{2} \pi \nu \sin \pi \nu
\end{aligned}
$$


and the asymptotic behavior

$$
\tilde{K}_{n}^{ \pm}(\underline{\theta}) \approx \pm 2 i \sin \pi \nu \tilde{K}_{n-1}^{ \pm}\left(\underline{\theta}^{\prime}\right) .
$$

The proof of the last formula is delegated to the appendix. The normalization constants are calculated analogously to the case of the general exponential and take the form

$$
N_{n}^{ \pm}= \pm i \frac{\sin \pi \nu}{\sin \frac{1}{2} \pi \nu}\left(\sqrt{Z^{\varphi}} \frac{\beta}{2 \pi \nu}\right)^{n} .
$$

\subsection{Identities}

It turns out that the correspondence between local operators and p-functions is not unique. In this subsection we prove some identities which we will need in the following section to prove operator equations. To have a consistent interpretation of $K_{n}^{(q)}(\underline{\theta})$ with $q=\exp (i \pi \nu \gamma / \beta)$ as the K-function of $\exp (i \gamma \varphi(x))$ it is necessary that $K_{n}^{(q)}(\underline{\theta})$ is even/odd for $n=$ even/odd under the exchange $q \leftrightarrow 1 / q$. For $\gamma= \pm \beta$ the K-function of the general exponential should turn into the $\mathrm{K}$-functions of the special exponentials. These fact are expressed by the following lemma.

Lemma 4 Let the K-functions

$$
K_{n}(\underline{\theta})=\sum_{l_{1}=0}^{1} \cdots \sum_{l_{r}=0}^{1}(-1)^{l_{1}+\cdots+l_{r}} \prod_{1 \leq i<j \leq n}\left(1+\left(l_{i}-l_{j}\right) \frac{i \sin \pi \nu}{\sinh \theta_{i j}}\right) p_{n}(\underline{\theta}, \underline{l})
$$

be given by the p-functions

$$
\begin{array}{r}
K_{n}^{(q)}(\underline{\theta}) \leftrightarrow p_{n}^{(q)}(\underline{l})=N_{n}^{(q)} \prod_{i=1}^{n} q^{(-1)^{l_{i}}} \\
K_{n}^{ \pm}(\underline{\theta}) \leftrightarrow p_{n}^{ \pm}(\underline{\theta}, \underline{l})=N_{n}^{ \pm} \sum_{i=1}^{n} e^{\mp \theta_{i}} \sum_{i=1}^{n} e^{ \pm\left(\theta_{i}-\frac{i \pi}{2}\left(1-(-1)^{l_{i}} \nu\right)\right)} \\
K_{n}^{(1)}(\underline{\theta}) \leftrightarrow p_{n}^{(1)}(\underline{l})=N_{n}^{(1)} \sum_{i=1}^{n}(-1)^{l_{i}} .
\end{array}
$$

Then the following identities hold (again with $K_{n}(\underline{\theta})=N_{n} \tilde{K}_{n}(\underline{\theta})$ )

$$
\begin{gathered}
\tilde{K}_{n}^{(q)}(\underline{\theta})=-(-1)^{n} \tilde{K}_{n}^{(1 / q)}(\underline{\theta}) \\
\tilde{K}_{n}^{+}(\underline{\theta})=-(-1)^{n} \tilde{K}_{n}^{-}(\underline{\theta}),
\end{gathered}
$$

in particular for $\gamma=\beta$ i.e. $q=\exp (i \pi \nu)$

$$
K_{n}^{+}(\underline{\theta})=K_{n}^{(q)}(\underline{\theta}) .
$$

and further more

$$
\tilde{K}_{n}^{(1)}(\underline{\theta})=\frac{1}{2 \sin \frac{1}{2} \pi \nu}\left(\sum_{i=1}^{n} e^{\theta_{i}} \sum_{i=1}^{n} e^{-\theta_{i}}\right)^{-1}\left(\tilde{K}_{n}^{+}(\underline{\theta})+\tilde{K}_{n}^{-}(\underline{\theta})\right) .
$$


Proof. Again as in the proof of lemma 2 we use induction and Liouville's theorem. We introduce the differences

$$
\begin{aligned}
f_{n}(\underline{\theta}) & =\tilde{K}_{n}^{(q)}(\underline{\theta})+(-1)^{n} \tilde{K}_{n}^{(1 / q)}(\underline{\theta}) \\
\text { or } f_{n}(\underline{\theta}) & =\tilde{K}_{n}^{+}(\underline{\theta})+(-1)^{n} \tilde{K}_{n}^{-}(\underline{\theta}) \\
\text { or } f_{n}(\underline{\theta}) & =K_{n}^{+}(\underline{\theta})-K_{n}^{(q=\exp (i \pi \nu))}(\underline{\theta}) \\
\text { or } f_{n}(\underline{\theta}) & =\tilde{K}_{n}^{(1)}(\underline{\theta})-\frac{1}{2 \sin \frac{1}{2} \pi \nu}\left(\sum_{i=1}^{n} e^{\theta_{i}} \sum_{i=1}^{n} e^{-\theta_{i}}\right)^{-1}\left(\tilde{K}_{n}^{+}(\underline{\theta})+\tilde{K}_{n}^{-}(\underline{\theta})\right) .
\end{aligned}
$$

Then the results of the previous subsection imply in all cases $f_{1}(\theta)=f_{2}(\underline{\theta})=$ 0 . As induction assumptions we take $f_{n-2}\left(\underline{\theta}^{\prime \prime}\right)=0$. The functions $f_{n}(\underline{\theta})$ are meromorphic functions in terms of the $x_{i}=e^{\theta_{i}}$ with at most simple poles at $x_{i}= \pm x_{j} \operatorname{since} \sinh \theta_{i j}=\left(x_{i}+x_{j}\right)\left(x_{i}-x_{j}\right) /\left(2 x_{i} x_{j}\right)$. The residues of the poles at $x_{i}=x_{j}$ vanish because of the symmetry and again the residues at $x_{i}=-x_{j}$ are proportional to $f_{n-2}\left(\underline{\theta}^{\prime \prime}\right)$ due to the recursion relation (11). Furthermore for $x_{i} \rightarrow \infty$ again $f_{n}(\underline{\theta}) \rightarrow 0$. Therefore $f_{n}(\underline{\theta})$ vanishes identically by Liouville's theorem in for all $n$. For the last case of $f_{n}(\underline{\theta})$ it has been used that because of (35) for $n$ even both $\tilde{K}^{ \pm}$-terms cancel and that they are equal for odd $n$. Due to (33) $\tilde{K}_{n}^{+}$is proportional to $\sum_{i=1}^{n} e^{-\theta_{i}}$ and $\tilde{K}_{n}^{-}$is proportional to $\sum_{i=1}^{n} e^{\theta_{i}}$. Hence both are proportional to $\sum_{i=1}^{n} e^{-\theta_{i}} \sum_{i=1}^{n} e^{+\theta_{i}}$ which means that there are no extra poles at $\sum_{i=1}^{n} e^{\theta_{i}}=0$ or $\sum_{i=1}^{n} e^{-\theta_{i}}=0$.

\section{Operator equations}

The classical sine-Gordon model is given by the wave equation

$$
\square \varphi(t, x)+\frac{\alpha}{\beta} \sin \beta \varphi(t, x)=0 .
$$

If this is fulfilled we have also the relation for the trace of energy-momentum tensor

$$
T_{\mu}^{\mu}(t, x)=-2 \frac{\alpha}{\beta^{2}}(\cos \beta \varphi(t, x)-1) .
$$

In this section we construct the quantum version of these two classical equations. In the following : $\cdots$ : denotes normal ordering with respect to the physical vacuum which means in particular for the vacuum expectation value $\langle 0|: \exp i \gamma \varphi:(t, x)| 0\rangle=1$. As consequences of the identities of subsection 4.4 we can prove quantum field equations.

Theorem 5 The following operator equations are to be understood in term of all their matrix elements.

1. For the exceptional value $\gamma=\beta$ the operator $\square^{-1}: \sin \gamma \varphi:(t, x)$ is local 
and the quantum sine-Gordon field equation holds $\$$

$$
\square \varphi(t, x)+\frac{\alpha}{\beta}: \sin \beta \varphi:(t, x)=0
$$

if the "bare" mass $\sqrt{\alpha}$ is related to the renormalized one by"

$$
\alpha=m^{2} \frac{\pi \nu}{\sin \pi \nu} .
$$

Here $m$ is the physical mass of the fundamental boson.

2. The energy momentum tensor is symmetric and its trace satisfies [0]

$$
T_{\mu}^{\mu}(t, x)=-2 \frac{\alpha}{\beta^{2}}\left(1-\frac{\beta^{2}}{8 \pi}\right)(: \cos \beta \varphi:(t, x)-1) .
$$

3. For all higher currents the conservation laws hold

$$
\partial_{\mu} J_{L}^{\mu}(x)=0 \quad(L= \pm 1, \pm 3, \ldots) .
$$

\section{Proof.}

1. From (33) we have the correspondence between operators and K-functions

$$
\square^{-1} \sin \beta \varphi \leftrightarrow \frac{K_{n}^{+}(\underline{\theta})-K_{n}^{-}(\underline{\theta})}{2 i \sum_{i=1}^{n} e^{\theta_{i}} \sum_{i=1}^{n} e^{-\theta_{i}}} .
$$

As shown in the proof of lemma there are no poles at $\sum_{i=1}^{n} e^{\theta_{i}}=0$ or $\sum_{i=1}^{n} e^{-\theta_{i}}=0$. Therefore $\square^{-1}: \sin \beta \varphi:$ is a local operator. Furthermore by eq. (36)

$$
\sum_{i=1}^{n} e^{\theta_{i}} \sum_{i=1}^{n} e^{-\theta_{i}} K_{n}^{(1)}(\underline{\theta})=\frac{\pi \nu}{\beta \sin \pi \nu} \frac{1}{2 i}\left(K_{n}^{+}(\underline{\theta})-K_{n}^{-}(\underline{\theta})\right)
$$

where the normalizations (27) and (34) have been used. This means in particular

$$
\frac{N_{n}^{(1)}}{N_{n}^{+}} \frac{i}{\sin \frac{1}{2} \pi \nu}=\frac{\pi \nu}{\beta \sin \pi \nu} .
$$

In terms of operators this is just the quantum sine-Gordon field equation. Comparing this result with the classical equation we obtain the relation eq. (38) between the bare and the physical mass.

\footnotetext{
${ }^{8}$ This field equation was also discussed in 17 and for the sinh-Gordon case in 22 . However, in these articles the relations of the bare and the renormalized mass differs from (38) and are not consistent with perturbation theoryand the results of [34, 35.

${ }^{9}$ Before this formula was found in 34 35] by different methods.

${ }^{10}$ This equation was also obtained by Smirnov 117 .
} 
2. Using (30) and (33) we have the correspondence between operators and K-functions for $n$ even

$$
\begin{aligned}
T^{+-} & \leftrightarrow N_{n}^{(T)} \tilde{K}_{n}^{-} \\
T^{-+} & \leftrightarrow-N_{n}^{(T)} \tilde{K}_{n}^{+} \\
T_{\mu}^{\mu} & \leftrightarrow K_{n}^{(T)}(\underline{\theta})=-\frac{1}{2} N_{n}^{(T)}\left(\tilde{K}_{n}^{+}-\tilde{K}_{n}^{-}\right) \\
\cos \beta \varphi-1 & \leftrightarrow \frac{1}{2}\left(K_{n}^{+}(\underline{\theta})+K_{n}^{-}(\underline{\theta})\right)
\end{aligned}
$$

The symmetry $T^{+-}=T^{-+}$is again a consequence of (35). Furthermore the identity of K-functions follows

$$
K_{n}^{(T)}(\underline{\theta})=-\frac{\alpha\left(1-\frac{\beta^{2}}{8 \pi}\right)}{\beta^{2}}\left(K_{n}^{+}(\underline{\theta})+K_{n}^{-}(\underline{\theta})\right)
$$

where the normalizations (32) and (34) have been used which means

$$
\frac{N_{n}^{(T)}}{N_{n}^{+}}=2 \frac{\alpha}{\beta^{2}}\left(1-\frac{\beta^{2}}{8 \pi}\right)
$$

3. The claim follows since we have the correspondence of operators and pfunctions

$$
\begin{aligned}
& \partial_{\mu} J_{L}^{\mu} \leftrightarrow P^{+} p_{n}^{(L,-)}(\underline{\theta}, \underline{l})+P^{-} p_{n}^{(L,+)}(\underline{\theta}, \underline{l})=-N_{n}^{\left(J_{L}\right)} m \\
& \quad \times\left(\sum_{i=1}^{n} e^{\theta_{i}} \sum_{i=1}^{n} e^{-\theta_{i}}-\sum_{i=1}^{n} e^{-\theta_{i}} \sum_{i=1}^{n} e^{\theta_{i}}\right) \sum_{i=1}^{n} e^{L\left(\theta_{i}-\frac{i \pi}{2}\left(1-(-1)^{l_{i}} \nu\right)\right)}=0 .
\end{aligned}
$$

The factor $\frac{\pi \nu}{\sin \pi \nu}$ modifies the classical equation and has to be considered as a quantum correction. For the sinh-Gordon model an analogous quantum field equation has been obtained in $[22]^{11}$. Note that in particular at the 'free fermion point' $\nu \rightarrow 1\left(\beta^{2} \rightarrow 4 \pi\right)$ this factor diverges, a phenomenon which is to be expected from short distance investigations [36]. For fixed bare mass square $\alpha$ and $\nu \rightarrow 2,3,4, \ldots$ the physical mass goes to zero. These values of the coupling are known to be special: 1. the Bethe Ansatz vacuum in the language of the massive Thirring model shows phase transitions [37] and 2. the model at these points is related [38, 39, 40] to Baxters RSOS-models which correspond to minimal conformal models with central charge $c=1-6 /(\nu(\nu+1))$ (see also [22]).

The second formula (39) is consistent with renormalization group arguments [41, 42]. In particular this means that $\beta^{2} / 4 \pi$ is the anomalous dimension of

\footnotetext{
${ }^{11}$ It should be obtained from (37) by the replacement $\beta \rightarrow i g$. However the relation petween the bare and the renormalized mass in 22. differs from the analytic continuation of 38.
} 
$\cos \beta \varphi$. Again this operator equation is modified by a quantum correction ( $1-$ $\beta^{2} / 8 \pi$ ). Obviously for fixed bare mass square $\alpha$ and $\beta^{2} \rightarrow 8 \pi$ the model will become conformal invariant. This in turn is related to a Berezinski-KosterlitzThouless phase transition [9, 43, 44]. The conservation law $\partial_{\mu} T^{\mu \nu}=0$ for the energy momentum tensor holds, because it is obtained from the higher currents for $L= \pm 1$. All the results may be checked in perturbation theory by Feynman

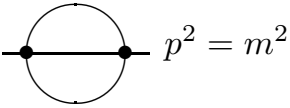

(a)

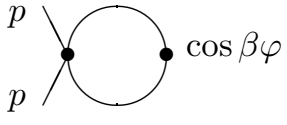

(b)

Figure 2: Feynman graphs

graph expansions. In particular in lowest order the relation between the bare and the renormalized mass (38) is given by figure 2 (a). It had already been calculated in 14] and yields

$$
m^{2}=\alpha\left(1-\frac{1}{6}\left(\frac{\beta^{2}}{8}\right)^{2}+O\left(\beta^{6}\right)\right)
$$

which agrees with the exact formula above. Similarly we check the quantum corrections of the trace of the energy momentum tensor (39) by calculating the Feynman graph of Figure 2 (b) with the result again taken from [14] as

$$
\langle p|: \cos \beta \varphi-1:| p\rangle=-\beta^{2}\left(1+\frac{\beta^{2}}{8 \pi}\right)+O\left(\beta^{6}\right) .
$$

This again agrees with the exact formula above since the normalization given by eq. (31) implies $\left\langle p\left|T_{\mu}^{\mu}\right| p\right\rangle=2 m^{2}$.

\section{Other representations of form factors}

\subsection{Determinant representation of bosonic sine-Gordon form factors}

The scaling Lee-Yang model is equivalent to the breather part of the sine-Gordon model for the coupling constant equal to $\nu=1 / 3$ (in our notation). For this model Smirnov [17] derived a determinant formula for form factors (see also [19]). Generalizing this formula in [20, 21] form factors were proposed for the sinh-Gordon model in terms of determinants. The sinh-Gordon model form factors should be identified with sine-Gordon form factors by analytic continuation $\nu \rightarrow$ negative values. Using this one would propose for the sine-Gordon model an analogous determinant representation for the K-function of exponentials of the field $\mathcal{O}=: e^{i k \beta \varphi}$ :

$$
\tilde{K}_{n}^{(q)}(\underline{\theta})=\left(q^{\prime}-1 / q^{\prime}\right)^{n} \prod_{i, j=1}^{n}\left(x_{i}+x_{j}\right)^{-1} \operatorname{Det}_{n}(\underline{x}, k)
$$


where $\underline{x}=\left(x_{1}, \ldots, x_{n}\right)$ with $x_{i}=e^{\theta_{i}}$ and $q^{\prime}=\exp i \pi \nu$. The Determinant is

$$
\begin{aligned}
\operatorname{Det}_{n}(\underline{x}, k) & =\operatorname{det}\left(\left((k+i-j)_{q^{\prime}} \sigma_{2 i-j-1}(\underline{x})\right)_{i, j=1}^{n}\right) \\
& =\left|\begin{array}{ccc}
(k)_{q^{\prime}} \sigma_{0} & \cdots & (k-n+1)_{q^{\prime}} \sigma_{-n+1} \\
\vdots & \ddots & \vdots \\
(k+n-1)_{q^{\prime}} \sigma_{2 n-2} & \cdots & (k)_{q^{\prime}} \sigma_{n-1}
\end{array}\right| .
\end{aligned}
$$

where the symmetric polynomials $\sigma_{l}(\underline{x})$ are defined by

$$
\prod_{l=1}^{n}\left(y+x_{l}\right)=\sum_{\lambda=0}^{n} y^{n-\lambda} \sigma_{\lambda}^{(n)}(\underline{x})
$$

and $(k)_{q^{\prime}}=\sin k \pi \nu / \sin \pi \nu$. This relation of $\tilde{K}_{n}^{(q)}(\underline{\theta})$ with $\operatorname{Det}_{n}(\underline{x}, k)$ could be proven similar as in the proof of lemma, , once the corresponding recursion relation (11) has been proven for $\operatorname{Det}_{n}(\underline{x}, k)$. This has been done 20] only for the special values $\nu=-1 / 2,-1 / 3$ of the coupling constant.

\subsection{Integral representations of breather form factors}

In [1] and [2] integral representations for solitonic form factors were proposed. These formulae are quite general and model independent, so analogous formulae should also hold for breather form factors. We propose for $n$ lowest breathers and $0 \leq m \leq n$

$$
\mathcal{O}_{n}(\underline{\theta})=\int_{\mathcal{C}_{\underline{\theta}}} d z_{1} \cdots \int_{\mathcal{C}_{\underline{\theta}}} d z_{m} h(\underline{\theta}, \underline{z}) \check{p}^{\mathcal{O}}(\underline{\theta}, \underline{z})
$$

with the scalar function (c.f. [1])

$$
h(\underline{\theta}, \underline{z})=\prod_{1 \leq i<j \leq n} F\left(\theta_{i j}\right) \prod_{i=1}^{n} \prod_{j=1}^{m} \tilde{\phi}\left(\theta_{i}-z_{j}\right) \prod_{1 \leq i<j \leq m} \tau\left(z_{i}-z_{j}\right)
$$

and

$$
\begin{gathered}
\tilde{\phi}(z)=\frac{S(z)}{F(z) F(z+i \pi)}=1+\frac{i \sin \pi \nu}{\sinh z}=\frac{\sinh z+i \sin \pi \nu}{\sinh z} . \\
\tau(z)=\frac{1}{\tilde{\phi}(z) \tilde{\phi}(-z)}=\frac{\sinh ^{2} z}{\sinh ^{2} z+\sin ^{2} \pi \nu} .
\end{gathered}
$$

The two breather form factor function $F(\theta)$ is again defined by eq. (价. For all integration variables $z_{j}(j=1, \ldots, m)$ the integration contours $\mathcal{C}_{\underline{\theta}}$ encloses clock wise oriented the points $z_{j}=\theta_{i}(i=1, \ldots, n)$. The above integral representation satisfies all form factor properties if suitable conditions for the new type of pfunction $12 \check{p}^{\mathcal{O}}(\theta, z)$ are assumed. Here we consider $\check{p}=$ constant. The form

\footnotetext{
${ }^{12}$ A similar function was used by Cardy and Mussardo 18 in case of the scaling Ising model to represent the various operators.
} 
factors of the exponential of the field $\mathcal{O}(x)=: \exp i \gamma \varphi:(x)$ are given by linear combinations of expressions (40)

$$
\mathcal{O}_{n}(\underline{\theta})=\left(\sqrt{Z^{\varphi}} \frac{\beta}{2 \pi \nu}\right)^{n} \prod_{1 \leq i<j \leq n} F\left(\theta_{i j}\right) \sum_{m=0}^{n} q^{n-2 m}(-1)^{m} I_{n m}(\underline{\theta})
$$

where again $q=\exp \left(i \frac{\pi \nu}{\beta} \gamma\right)$ and

$$
\begin{aligned}
I_{n m}(\underline{\theta}) & =\frac{1}{m !} \int_{\mathcal{C}_{\underline{\theta}}} \frac{d z_{1}}{R} \cdots \int_{\mathcal{C}_{\underline{\theta}}} \frac{d z_{m}}{R} \prod_{i=1}^{n} \prod_{j=1}^{m} \tilde{\phi}\left(\theta_{i}-z_{j}\right) \prod_{1 \leq i<j \leq m} \tau\left(z_{i}-z_{j}\right) \\
& =\sum_{\substack{K \subseteq N \\
|K|=m}} \prod_{i \in N \backslash K} \prod_{k \in K} \tilde{\phi}\left(\theta_{i k}\right)
\end{aligned}
$$

with $R=\operatorname{Res}_{\theta=0} \tilde{\phi}(\theta)$ and $N=\{1, \ldots, n\}, K=\left\{k_{1}, \ldots, k_{m}\right\}$. It is easy to verify that the asymptotic behavior (20) is satisfied. Also the low particle number form factors agree with eqs. (24). This proves formula (41).

Another integral representation is directly obtained from the integral representations for solitonic form factors in [2]

$$
\begin{aligned}
\mathcal{O}(\underline{\theta})=\tilde{N}_{n}^{\mathcal{O}} & \prod_{i<j}\left(F\left(\theta_{i j}\right) \tanh \frac{1}{2} \theta_{i j} \sinh \frac{1}{2}\left(\theta_{i j}+i \pi \nu\right) \sinh \frac{1}{2}\left(\theta_{i j}-i \pi \nu\right)\right) \\
& \times \int_{\mathcal{D}_{\theta_{1}}} d z_{1} \cdots \int_{\mathcal{D}_{\theta_{n}}} d z_{r} \prod_{i=1}^{n} \prod_{j=1}^{n} \chi\left(\theta_{i}-z_{j}\right) \prod_{i<j} \sinh z_{i j} p(\underline{\theta}, \underline{z})
\end{aligned}
$$

where the contour $\mathcal{D}_{\theta_{i}}$ consists of two circles around the poles at $\theta_{i}-\frac{i \pi}{2}(1 \pm \nu)$ and

$$
\chi(\theta)=\frac{1}{\sinh \frac{1}{2}\left(\theta-\frac{i \pi}{2}(1-\nu)\right) \sinh \frac{1}{2}\left(\theta-\frac{i \pi}{2}(1+\nu)\right)} .
$$

As a matter of fact in 2 from this integral representation the representation (1) with the K-function (2) was derived using the identity

$$
\begin{aligned}
\sinh & \frac{1}{2}\left(\theta_{i j}-i \pi \nu\right) \chi\left(\theta_{i}-z_{j}^{\left(l_{j}\right)}\right) \chi\left(\theta_{j}-z_{i}^{\left(l_{i}\right)}\right) \sinh \left(z_{i}^{\left(l_{i}\right)}-z_{j}^{\left(l_{j}\right)}\right) \\
& =\frac{2}{\tanh \frac{1}{2} \xi_{i j} \sinh \frac{1}{2}\left(\xi_{i j}+i \pi \nu\right) \sinh \frac{1}{2}\left(\xi_{i j}-i \pi \nu\right)}\left(1+\left(l_{i}-l_{j}\right) \frac{i \sin \pi \nu}{\sinh \xi_{i j}}\right)
\end{aligned}
$$

for $l_{i}, l_{j}=0,1$ and $z_{i}^{\left(l_{i}\right)}=\xi_{i}-\frac{i \pi}{2}\left(1-(-1)^{l_{i}} \nu\right)$. Performing one integration in eq. (42) and using symmetry properties of the integrand one obtains an integral representations of the type as used by Smirnov in [17].

Acknowledgments: We thank J. Balog, A.A. Belavin, V.A. Fateev, R. Flume, A. Fring, S. Pakuliak, R.H. Poghossian, R. Schrader, B. Schroer, F.A. 
Smirnov and Al.B. Zamolodchikov for discussions. One of authors (M.K.) thanks E. Seiler and P. Weisz for discussions and hospitality at the Max-Planck Insitut für Physik (München), where parts of this work have been performed. H.B. was supported by DFG, Sonderforschungsbereich 288 'Differentialgeometrie und Quantenphysik' and partially by grants INTAS 99-01459 and INTAS 00-561.

\section{Appendix: Asymptotic behavior}

Lemma 6 The K-functions defined by eq. (2) and the p-functions

$$
\begin{array}{ll}
\text { a) } & \tilde{p}_{n}^{(q)}(\underline{\theta})=\prod_{i=1}^{n} q^{(-1)^{l_{i}}} \\
\text { b) } & \tilde{p}_{n}^{(N)}(\underline{\theta})=\left(\sum_{i=1}^{n}(-1)^{l_{i}}\right)^{N} \\
\text { c) } & \tilde{p}_{n}^{( \pm)}(\underline{\theta})=\sum_{i=1}^{n} e^{\mp \theta_{i}} \sum_{i=1}^{n} e^{ \pm z_{i}^{\left(l_{i}\right)}}
\end{array}
$$

satisfy for $\operatorname{Re} \theta_{1} \rightarrow \infty$ the asymptotic behavior

$$
\begin{array}{ll}
\text { a) } & \tilde{K}_{n}^{(q)}(\underline{\theta})=\tilde{K}_{1}^{(q)}\left(\theta_{1}\right) \tilde{K}_{n-1}^{(q)}\left(\underline{\theta}^{\prime}\right)+O\left(e^{-\operatorname{Re} \theta_{1}}\right) \\
\text { b) } & \tilde{K}_{n}^{(N)}(\underline{\theta})=\sum_{K=1}^{N-1}\left(\begin{array}{l}
N \\
K
\end{array}\right) \tilde{K}_{1}^{(K)}\left(\theta_{1}\right) \tilde{K}_{n-1}^{(N-K)}\left(\underline{\theta}^{\prime}\right)+O\left(e^{-\operatorname{Re} \theta_{1}}\right) \\
\text { c) } & \tilde{K}_{n}^{ \pm}(\underline{\theta})= \pm 2 i \sin \pi \nu \tilde{K}_{n-1}^{ \pm}\left(\underline{\theta}^{\prime}\right)+O\left(e^{-\operatorname{Re} \theta_{1}}\right)
\end{array}
$$

with $\underline{\theta}^{\prime}=\left(\theta_{2}, \ldots, \theta_{1}\right)$. In particular

$$
\tilde{K}_{1}^{(1)}(\theta)=\text { const } \quad \text { and } \quad \tilde{K}_{n}^{(1)}(\underline{\theta})=O\left(e^{-\operatorname{Re} \theta_{1}}\right) \quad \text { for } n>1 \text {. }
$$

Proof. The two first asymptotic relations are quite obvious. Note that $\tilde{K}_{1}^{(q)}=$ $q-1 / q$ and $\tilde{K}_{1}^{(1)}(\underline{\theta})=2$.

a) For $\tilde{K}_{n}^{(q)}(\underline{\theta})$ and $\operatorname{Re} \theta_{1} \rightarrow \infty$ we have

$$
\tilde{K}_{n}^{(q)}(\underline{\theta})=\sum_{l_{1}=0}^{1}(-1)^{l_{1}} q^{(-1)^{l_{1}}} \tilde{K}_{n-1}^{(q)}\left(\underline{\theta}^{\prime}\right)+O\left(e^{-\operatorname{Re} \theta_{1}}\right)
$$

b) For $\tilde{K}_{n}^{(N)}(\underline{\theta})$ we use

$$
\left(\sum_{i=1}^{n}(-1)^{l_{i}}\right)^{N}=\sum_{K=0}^{N}\left(\begin{array}{l}
N \\
K
\end{array}\right)\left((-1)^{l_{1}}\right)^{K}\left(\sum_{i=2}^{n}(-1)^{l_{i}}\right)^{N-K}
$$

which proves the claim as in the previous case. 
c) For $\tilde{K}_{n}^{+}(\underline{\theta})$ and $\operatorname{Re} \theta_{1} \rightarrow \infty$ we have

$$
\begin{array}{r}
\sum_{i=1}^{n} e^{-\theta_{i}} \sum_{i=1}^{n} e^{z_{i}^{\left(l_{i}\right)}}=\left(e^{-\theta_{1}}+\sum_{i=2}^{n} e^{-\theta_{i}}\right)\left(e^{\theta_{1}-\frac{i \pi}{2}\left(1-(-1)^{l_{1}} \nu\right)}+\sum_{i=2}^{n} e^{z_{i}^{\left(l_{i}\right)}}\right) \\
=e^{-\frac{i \pi}{2}\left(1-(-1)^{l_{1}} \nu\right)}+\left(\sum_{i=2}^{n} e^{-\theta_{i}}\right) e^{\theta_{1}-\frac{i \pi}{2}\left(1-(-1)^{l_{1}} \nu\right)} \\
+\sum_{i=2}^{n} e^{-\theta_{i}} \sum_{i=2}^{n} e^{z_{i}^{\left(l_{i}\right)}}+O\left(e^{-\operatorname{Re} \theta_{1}}\right) .
\end{array}
$$

We calculate the leading terms $O(1)$. The contribution of the first term consists of two types: one vanishes because of the lemma above and the other is of order $O\left(e^{-\operatorname{Re} \theta_{1}}\right)$. The contribution of the third term vanishes after summation over $l_{1}$. The contribution of the second term is proportional to

$$
\begin{aligned}
& \sum_{l_{1}=0}^{1}(-1)^{l_{1}}\left(1+\sum_{j=2}^{n}\left(l_{1}-l_{j}\right) \frac{i \sin \pi \nu}{\sinh \theta_{1 j}}\right) e^{\theta_{1}-\frac{i \pi}{2}\left(1-(-1)^{l_{1}} \nu\right)} \\
& \approx-i e^{\theta_{1}}\left(e^{\frac{i \pi}{2} \nu}-e^{-\frac{i \pi}{2} \nu}\right)+2 i \sin \pi \nu \sum_{j=2}^{n} \sum_{l_{1}=0}^{1}(-1)^{l_{1}}\left(l_{1}-l_{j}\right) e^{\theta_{j}-\frac{i \pi}{2}\left(1-(-1)^{l_{1}} \nu\right)}
\end{aligned}
$$

The first term again vanishes due to the lemma and the second one yields

$$
\begin{aligned}
& 2 i \sin \pi \nu \sum_{j=2}^{n} \sum_{l_{1}=0}^{1}(-1)^{l_{1}}\left(l_{1}-l_{j}\right) e^{\theta_{j}-\frac{i \pi}{2}\left(1-(-1)^{l_{1}} \nu\right)} \\
& =2 i \sin \pi \nu \sum_{j=2}^{n} \sum_{l_{1}=0}^{1}(-1)^{l_{1}}\left(l_{1}-l_{j}\right) e^{\theta_{j}-\frac{i \pi}{2}\left(1-(-1)^{1-l_{j}} \nu\right)} \\
& =-2 i \sin \pi \nu \sum_{j=2}^{n} e^{\theta_{j}-\frac{i \pi}{2}\left(1+(-1)^{l_{j}} \nu\right)}
\end{aligned}
$$

Therefore we finally obtain the asymptotic behavior

$$
\begin{aligned}
\tilde{K}_{n}^{+}(\underline{\theta})= & -2 i \sin \pi \nu \sum_{l_{2}=0}^{1} \cdots \sum_{l_{n}=0}^{1}(-1)^{l_{2}+\cdots+l_{n}} \\
& \times \prod_{2 \leq i<j \leq n}\left(1+\left(l_{i}-l_{j}\right) \frac{i \sin \pi \nu}{\sinh \theta_{i j}}\right) \\
& \times \sum_{i=2}^{n} e^{-\theta_{i}} \sum_{j=2}^{n} e^{\theta_{j}-\frac{i \pi}{2}\left(1+(-1)^{l_{j}} \nu\right)}+O\left(e^{-\operatorname{Re} \theta_{1}}\right) \\
= & 2 i \sin \pi \nu \tilde{K}_{n-1}^{+}\left(\underline{\theta^{\prime}}\right)+O\left(e^{-\operatorname{Re} \theta_{1}}\right)
\end{aligned}
$$


with $\underline{\theta^{\prime}}=\left(\theta_{2}, \ldots, \theta_{n}\right)$. We have used

$$
\begin{aligned}
\sum_{l_{2}=0}^{1} \cdots \sum_{l_{n}=0}^{1}(-1)^{l_{2}+\cdots+l_{n}} & \prod_{2 \leq i<j \leq n}\left(1+\left(l_{i}-l_{j}\right) \frac{i \sin \pi \nu}{\sinh \theta_{i j}}\right) \\
& \times \sum_{j=2}^{n}\left(e^{\theta_{j}-\frac{i \pi}{2}\left(1+(-1)^{l_{j}} \nu\right)}+e^{\theta_{j}-\frac{i \pi}{2}\left(1-(-1)^{l_{j}} \nu\right)}\right)=0
\end{aligned}
$$

which follows from lemma 2. For $\tilde{K}_{n}^{-}(\underline{\theta})$ and $\operatorname{Re} \theta_{1} \rightarrow \infty$ we have

$$
\begin{aligned}
\sum_{i=1}^{n} e^{\theta_{i}} \sum_{i=1}^{n} e^{-z_{i}^{\left(l_{i}\right)}}= & \left(e^{\theta_{1}}+\sum_{i=2}^{n} e^{\theta_{i}}\right)\left(e^{-\theta_{1}+\frac{i \pi}{2}\left(1-(-1)^{l_{1}} \nu\right)}+\sum_{i=2}^{n} e^{-z_{i}^{\left(l_{i}\right)}}\right) \\
= & e^{\frac{i \pi}{2}\left(1-(-1)^{l_{1}} \nu\right)}+e^{\theta_{1}} \sum_{i=2}^{n} e^{-\theta_{j}-\frac{i \pi}{2}\left(1-(-1)^{l_{j}} \nu\right)} \\
& +\sum_{i=2}^{n} e^{\theta_{i}} \sum_{i=2}^{n} e^{-z_{i}^{\left(l_{i}\right)}}+O\left(e^{-\operatorname{Re} \theta_{1}}\right) .
\end{aligned}
$$

We again calculate the leading terms $O(1)$. Again the contribution of the first term consists of two types: one vanishes because of the lemma above and the other is of order $O\left(e^{-\operatorname{Re} \theta_{1}}\right)$. The contribution of the third term vanishes after summation over $l_{1}$. The contribution of the second term is proportional to

$$
\begin{aligned}
& \sum_{l_{1}=0}^{1}(-1)^{l_{1}}\left(1+\sum_{j=2}^{n}\left(l_{1}-l_{i}\right) \frac{i \sin \pi \nu}{\sinh \theta_{1 i}}\right) e^{\theta_{1}} \sum_{j=2}^{n} e^{-\theta_{j}+\frac{i \pi}{2}\left(1-(-1)^{l_{j}} \nu\right)} \\
& \approx 2 i \sin \pi \nu \sum_{j=2}^{n} e^{\theta_{i}} \sum_{l_{1}=0}^{1}(-1)^{l_{1}}\left(l_{1}-l_{j}\right) e^{-\theta_{j}+\frac{i \pi}{2}\left(1-(-1)^{l_{j}} \nu\right)} \\
& =-2 i \sin \pi \nu \sum_{j=2}^{n} e^{\theta_{i}} \sum_{j=2}^{n} e^{-\theta_{j}+\frac{i \pi}{2}\left(1-(-1)^{l_{j}} \nu\right)}
\end{aligned}
$$

Therefore we finally obtain the asymptotic behavior

$$
\begin{aligned}
\tilde{K}_{n}^{-}(\underline{\theta})= & -2 i \sin \pi \nu \sum_{l_{2}=0}^{1} \cdots \sum_{l_{n}=0}^{1}(-1)^{l_{2}+\cdots+l_{n}} \\
& \times \prod_{2 \leq i<j \leq n}^{n}\left(1+\left(l_{i}-l_{j}\right) \frac{i \sin \pi \nu}{\sinh \theta_{i j}}\right) \\
& \times \sum_{i=2}^{n} e^{\theta_{i}} \sum_{j=2}^{n} e^{-\theta_{j}+\frac{i \pi}{2}\left(1+(-1)^{l_{j}} \nu\right)}+O\left(e^{-\operatorname{Re} \theta_{1}}\right) \\
= & -2 i \sin \pi \nu \tilde{K}_{n-1}^{-}\left(\underline{\theta^{\prime}}\right)+O\left(e^{-\operatorname{Re} \theta_{1}}\right) .
\end{aligned}
$$

Analogously one may discuss the behavior for $\operatorname{Re} \theta_{1} \rightarrow-\infty$. 


\section{References}

[1] H. Babujian, A. Fring, M. Karowski and A. Zapletal, Nucl. Phys. B538 [FS] (1999) 535-586.

[2] H. Babujian and M. Karowski, Nucl. Phys. B620 (2002) 407-455.

[3] H.M. Babujian and M. Karowski, Phys. Lett. B 411 (1999) 53-57.

[4] S. Coleman, Phys. Rev. D11 (1975) 2088.

[5] F.A. Smirnov, Lett. Math. Phys. 36 (1996) 267-275;

Nucl. Phys. B253 (1995) 807-824.

[6] Y. Takeyama, Form factors of $S U(N)$ invariant Thirring model, math$\mathrm{ph} / 0112025$.

[7] M. Karowski, H.J. Thun, T.T. Troung, and P. Weisz, Phys. Lett. 67B (1977) 321.

[8] M. Karowski, The bootstrap program for $1+1$ dimensional field theoretic models with soliton behavior, in 'Field theoretic methods in particle physics', ed. W. Rühl, (Plenum Pub. Co., New York, 1980).

[9] M. Karowski and H.J. Thun, Nucl. Phys. B130 (1977) 295.

[10] S. Vergeles and V. Gryanik, Sov. Journ. Nucl. Phys. 23 (1976) 704.

[11] I.Ya. Arefyeva and V.E. Korepin, JETP Lett. 20 (1974) 312.

[12] P. Weisz, Phys. Lett. 67B (1977) 179.

[13] A.B. Zamolodchikov, Moscow preprint ITEP 45 (1977).

[14] M. Karowski and P. Weisz, Nucl. Phys. B139 (1978) 445.

[15] B. Berg, M. Karowski and P. Weisz, Phys. Rev. D19 (1979) 2477.

[16] F.A. Smirnov 'Form Factors in Completely Integrable Models of Quantum Field Theory', Adv. Series in Math. Phys. 14, World Scientific 1992.

[17] F.A. Smirnov, Nucl. Phys. B337 (1990) 156-180.

[18] J.L. Cardy and G. Mussardo, Phys. Lett. B225 (1989) 275. Nucl. Phys. B340 (1990) 387.

[19] Al.B. Zamolodchikov, Nucl. Phys. B348 (1991) 619-641.

[20] A. Fring, G. Mussardo and P. Simonetti, Nucl. Phys. B393 (1993) 413.

[21] A. Koubeck and G. Mussardo, Phys. Lett. B311 (1993) 193.

[22] G. Mussardo and P. Simonetti, Int. J. Mod. Phys. A9 (1994) 3307-3338. 
[23] V. Brazhnikov and S. Lukyanov,Nucl. Phys. B512 (1998) 616-636.

[24] S Lukyanov, Mod. Phys. Lett. A 12 (1997) 2543-2550.

[25] S Lukyanov and A.B. Zamolodchikov, Nucl. Phys. B607 (2001) 437.

[26] S. Khoroshkin, D. Lebedev and S. Pakuliak, Lett. Math. Phys. 41 (1997) 31-47.

[27] A. Nakayashiki, S. Pakuliak and V. Tarasov, Annales de l'Institut Henri Poincaré 71 N4 (1999) 459-496.

[28] O.A. Castro-Alvaredo and A. Fring, Nucl. Phys. B618 (2001) 437-464.

[29] A.O. Gogolin, A.A Nersesyan and A.M. Tsvelik, 'Bosonization in Strongly Correlated Systems', Cambridge University Press (1999).

[30] D. Controzzi, F.H.L. Essler and A.M. Tsvelik, 'Dynamical Properties of one dimensional Mott Insulators', to appear in the proceedings of the NATO ASI/EC summer school "New Theoretical Approaches to Strongly Correlated Systems" Newton Institute for Mathematical Sciences, Cambridge UK, April 2000.

[31] A. Fring, unpublished and private communication.

[32] R. Schrader, Fortschritte der Physik 22 (1974) 611-631.

[33] J. Frölich, in "Renormalization Theory", ed. G. Velo et al. (Reidel,1976) 371.

[34] V.A. Fateev, Phys. Lett. B324 (1994) 45-51.

[35] Al.B. Zamolodchikov, Int. J. Mod. Phys. A10 (1995) 1125-1150.

[36] B. Schroer and T. Truong, Phys. Rev. 15 (1977) 1684.

[37] V.E. Korepin, Commun. Math. Phys. 76 (1980) 165.

[38] M. Karowski, Nucl. Phys. B300 [FS22] (1988) 473; -, Yang-Baxter algebra - Bethe ansatz - conformal quantum field theories-quantum groups, in 'Quantum Groups', Lecture Notes in Physics, Springer (1990) 183

[39] A. LeClair, Phys. Lett. B230 (1989) 103-107

[40] F.A. Smirnov, Commun. Math. Phys. 131 (1990) 157-178.

[41] A.B. Zamolodchikov, JETP Lett. 43 (1986) 730; Nucl. Phys 46 (1987) 1090.

[42] J.L. Cardy, Phys. Rev. Lett. 60 (1988) 2709.

[43] J.Jose, L. Kadanoff, S. Kirkpatrick and D. Nelson, Phys. Rev B16 (1977) 1217.

[44] P.B. Wiegmann, J. Phys. C11 (1978) 1583. 